\title{
Rapid spectral evolution of steep surface wave groups with directional spreading
}

\author{
D. Barratt ${ }^{1} \dagger$, H. B. Bingham ${ }^{2}$, P. H. Taylor ${ }^{3}$, T. S. van den Bremer ${ }^{1}$ \\ and T. A. A. Adcock ${ }^{1}$ \\ ${ }^{1}$ Department of Engineering Science, University of Oxford, Oxford OX1 3PJ, UK \\ ${ }^{2}$ Department of Mechanical Engineering, Technical University of Denmark (DTU), \\ 2800 Lyngby, Denmark \\ ${ }^{3}$ Faculty of Engineering and Mathematical Sciences, University of Western Australia, \\ Crawley WA 6009, Australia \\ (Received $\mathrm{xx}$; revised $\mathrm{xx}$; accepted $\mathrm{xx}$ )
}

We have investigated steep three-dimensional surface gravity wave groups formed by dispersive focusing using a fully-nonlinear potential flow solver. We find that third-order resonant interactions result in rapid energy transfers to higher wavenumbers and reduced directional spreading during focusing, followed by spectral broadening during defocusing, forming steep wave groups with augmented kinematics and a prolonged lifespan. If the wave group is initially narrow-banded, quasi-degenerate interactions arise, characterised by energy transfers along the resonance angle, $\pm 35.26^{\circ}$, of the Phillips 'figureof-eight' loop. Spectral broadening due to the quasi-degenerate interactions facilitates non-degenerate interactions, characterised by oblique energy transfers at approximately $\pm 55^{\circ}$ to the spectral peak. We consider the influence of steepness, finite depth, directional spreading and the high-wavenumber tail on spectral evolution. Steepness is found to augment both the quasi-degenerate and non-degenerate interactions similarly. However, a reduction in depth is found to weaken the quasi-degenerate interactions more severely than the non-degenerate interactions. We observe that increased directional spreading reduces spectral evolution, partially because wave groups with more spreading focus for a shorter duration due to linear dispersion. However, we also find that directional spreading reduces the peak rates of energy transfer. Inclusion of the high-wavenumber tail of the JONSWAP spectrum further reduces rates of energy transfer compared with a Gaussian wavenumber spectrum. Thus, directional spreading and the high-wavenumber tail may be integral to a form of spectral equilibrium that reduces rapid energy transfers during a steep wave event.

\section{Introduction}

Steep groups of surface gravity waves can arise from a variety of mechanisms, both linear and nonlinear. Frequency and directional dispersion can facilitate constructive interference between wave components (see for example Fedele et al. (2016)) while nonlinear wave-wave interactions alter the dispersive characteristics of a wave field allowing for self focusing (Janssen 2003). Interactions with wind, current and bathymetry have been identified as further focusing mechanisms. Reviews by Kharif \& Pelinovsky (2003), Kharif et al. (2008b), Dysthe et al. (2008), and Adcock \& Taylor (2014) provide an extensive overview of steep wave formation and 'rogue waves'. The characteristics

$\dagger$ Email address for correspondence: dylan.barratt@eng.ox.ac.uk 

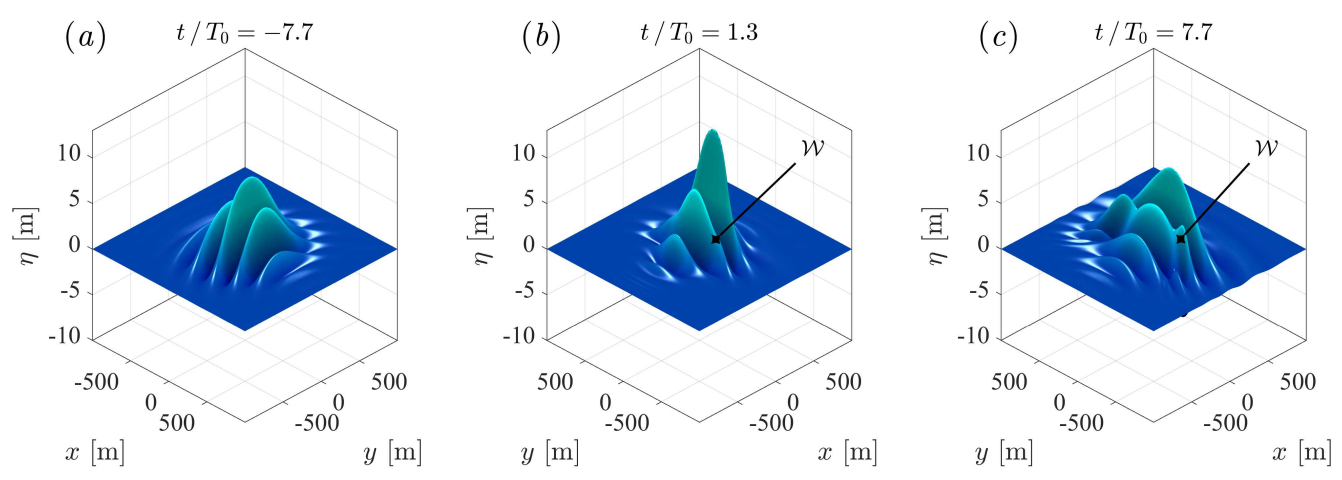

FIGURE 1. Surface elevation $(\eta)$ of a steep wave group formed by dispersive focusing, simulated by Barratt et al. (2020) with OceanWave3D: (a) pre-focus, $t / T_{0}=-7.5$; (b) nonlinear focus, $t / T_{0}=1.3$; (c) post-focus, $t / T_{0}=7.5$. Times are defined relative to the linear focus time, normalised by the characteristic period $\left(T_{0}\right)$. The formation of 'wing waves', localised regions of elevation due to energised oblique components, is demarcated with $\mathcal{W}$. These results have been compared against the HOS simulations of Gibbs \& Taylor (2005); the maximum surface elevation agrees within $0.3 \%$ with total energy conservation within $0.024 \%$ over 40 wave periods.

of steep wave groups have been investigated in numerous previous studies, both in the context of random seas and isolated wave groups.

The random sea approach, characterised by an initially-uniform phase distribution, captures the natural variability of wave focusing as well as interactions between neighbouring focused events and the global evolution of the background sea state. Steep wave events in a random sea have been found to exhibit front-rear asymmetry (Xiao et al. (2013), Adcock et al. (2015), Fujimoto et al. (2019)), contraction in the direction of propagation (Adcock et al. (2015)) and a localised reduction in spreading at focus (Adcock et al. (2015), Latheef et al. (2017)). Steep wave groups in a random sea have also been observed to exhibit a prolonged 'lifetime' due to nonlinear interactions (Fujimoto et al. (2019)). Random sea simulations and experiments are, however, innately inefficient because the vast majority of the observed wave events are not particularly steep. An alternative approach based on isolated wave groups has thus been pursued by previous studies, characterised by a coherent phase distribution designed to focus wave components in time and space. This is less computationally expensive, allows error waves associated with low-order numerical wave paddles or initial conditions to be easily identified and discounted (since the error waves separate out from the main group) and highlights changes in group shape. The wave-wave interactions which arise for an isolated wave group are driven by the same physical processes as those of a random sea, and we use the isolated wave group approach in this study. Numerical simulations of isolated wave groups performed by Gibbs \& Taylor (2005) as well as Gibson \& Swan (2007) reported 'rapid' changes to the underlying wave spectrum, altering the amplitude and phase speed of the resonant components, and attributed the spectral changes to third-order resonant interactions. Barratt et al. (2020) used the potential flow code Ocean Wave3D to simulate focusing wave groups, depicted before focus in figure 1(a), at nonlinear focus in figure $1(\mathrm{~b})$, and after focus in figure 1(c). Directional energy transfers were found to cause the formation of 'wing waves', localised oblique protrusions at the periphery of the wave group marked with $\mathcal{W}$ in figure 1 . The formation of wing waves has been attributed by Barratt et al. (2020) to third-order resonant interactions. Thus, previous studies indicate that third-order resonant interactions influence the characteristics of steep wave groups formed by dispersive focusing. 
Third-order resonance for deep-water waves has been shown by Phillips (1960) to occur between four wave components that satisfy the conditions:

$$
\boldsymbol{k}_{1} \pm \boldsymbol{k}_{2} \pm \boldsymbol{k}_{3} \pm \boldsymbol{k}_{4}=0,
$$

and with the same combination of signs,

$$
\omega_{1} \pm \omega_{2} \pm \omega_{3} \pm \omega_{4}=\delta \omega
$$

consistent with the deep-water linear dispersion relationship,

$$
\omega_{i}^{2}=g k_{i}
$$

Here, the wavenumber and angular frequency of each component are denoted with $\boldsymbol{k}_{i}$ and $\omega_{i}$, respectively. The gravitational constant is denoted with $g$, and $\delta \omega$ represents an angular frequency mismatch which detunes the interaction. Resonant interactions can be categorised as either exactly resonant, if $\delta \omega$ is strictly zero, or nearly resonant, if $\delta \omega$ is $O\left(\epsilon^{2} \omega_{0}\right)$, based on a characteristic frequency $\left(\omega_{0}\right)$. As discussed by Stiassnie (2017), the Zakharov equation accounts for exactly and nearly-resonant quartets, with an associated dynamic timescale of $O\left(\epsilon^{-2} T_{0}\right)$, based on the characteristic time scale of the wave field $\left(T_{0}\right)$. In contrast, the Hasselmann (1962) equation only accounts for exactly resonant quartets, with an associated kinetic timescale of $O\left(\epsilon^{-4} T_{0}\right)$. A quartet of interacting wave components is termed 'degenerate' if only three unique wave components are involvedone of the components participates twice in the interaction (e.g., $\boldsymbol{k}_{1}=\boldsymbol{k}_{2}$ ). In this study, we also consider the role of 'quasi-degenerate' quartets, characterised by $\boldsymbol{k}_{1} \approx \boldsymbol{k}_{2}$. Quasidegenerate quartets do not meet the strict definition of a degenerate quartet but can be expected to share certain characteristics which we analyse and discuss. We use the term 'non-degenerate' to describe quartet interactions characterised by $\boldsymbol{k}_{1} \neq \boldsymbol{k}_{2}$. Previous studies indicate that quartet interactions are particularly prevalent for narrow-banded wave fields and wave fields which are 'out of equilibrium', both of which are considered in this study.

Narrow-banded wave fields have been a particular focus of previous studies due to their large third-order interactions. Longuet-Higgins (1976) investigated the resonant interactions of a narrow-banded wave spectrum, using the Davey \& Stewartson (1974) equation, and calculated a resonance angle of $\arctan ( \pm 1 / \sqrt{2})= \pm 35.26^{\circ}$ relative to the spectral peak. Notably, the angle calculated by Longuet-Higgins (1976) matches the resonance angle of the Phillips (1960) 'figure-of-eight' loop for a degenerate quartet and the instability region of the 2+1 NLS equation, as calculated by Zakharov (1968), Benney \& Roskes (1969) and Davey \& Stewartson (1974). Crawford et al. (1981) used the Zakharov equation to investigate the stability of a wave train to three-dimensional modulations and observed instability bands contained within the Phillips resonance loop. The eigenvalue stability analysis of McLean (1982b) also revealed quartet instability bands within the Phillips resonance loop for a finite-amplitude regular wave and showed that the dominant quartet instabilities are unidirectional in deep water. Alber (1978) performed a stability analysis, based on the Davey \& Stewartson (1974) equation, and found that a homogeneous spectrum of random waves is unstable to oblique inhomogeneous disturbances at angles less than $\pm 35.26^{\circ}$ if the bandwidth is sufficiently narrow but becomes stable for broader bandwidths. Conversely, Stuhlmeier \& Stiassnie (2017) observed evidence of instability for broad-banded spectra subject to small inhomogeneous disturbances, using a discretized version of the Zakharov equation without bandwidth limits. In this study, our simulations of narrow-banded wave groups exhibit directional energy transfers which resemble the Philips resonance loop for a degenerate quartet. However, as the wave group becomes more broad-banded we observe other directional 
energy transfers not encompassed by the Phillips resonance loop. We discuss the impact of the spectral evolution on the shape, kinematics and lifespan of the wave groups and assess the effects of steepness, finite depth, directional spreading and the high-wavenumber tail.

Spectral equilibrium is another factor which has been linked by previous studies to the importance of third-order interactions. The role of wave-field equilibrium in the formation of extreme waves has been highlighted by Trulsen (2018); sudden changes in water depth and meteorological conditions were identified as possible causes for non-equilibrium. Random wave fields which are 'out of equilibrium' are expected to evolve on the dynamic $O\left(\epsilon^{-2} T_{0}\right)$ timescale, as discussed by Annenkov \& Shrira (2006) and Annenkov \& Shrira (2018). Onorato \& Suret (2016) identified wind, current and topography and factors which can bring a wave field out of equilibrium. As noted by Trulsen (2018), random wave-field simulations initialised with artificial spectra exhibit rapid spectral evolution during the early-stages of the simulation (see, e.g., Dysthe et al. (2003), Socquet-Juglard et al. (2005), Toffoli et al. (2010) and Xiao et al. (2013)). A similar effect has been observed experimentally by Shemer et al. (2010) for waves generated from an artificial spectrum in a laboratory tank. Gramstad \& Trulsen (2007) performed MNLS simulations based on the equation of Trulsen \& Dysthe (1996) and found that the occurrence of freak waves relates to crest and group length, highlighting the role of spectral bandwidth and spreading. The theory of wave turbulence associates nonlinear interactions (and non-Gaussian properties) with intermittency and Fadaeiazar et al. (2018) found that directional spreading reduces intermittency also indicating that spreading may be an important feature of spectral equilibrium. Various early studies have furthered the discussion of wave-field equilibrium, including: the wind speed investigation of Waseda et al. (2001), the NLS simulations of Onorato et al. (2001), the wave turbulence study of Onorato et al. (2002), the kurtosis evolution analysis of Janssen (2003) and the phase-resolved stochastic analysis of Stiassnie \& Shemer (2005). In this study, we perform simulations based on Gaussian as well as JONSWAP spectra, with different spreading functions, and consider the role of directional spreading and the high-wavenumber tail in spectral equilibrium for steep wave groups formed by dispersive focusing.

In summary, we perform numerical simulations of narrow-banded wave groups, formed by dispersive focusing, using a fully-nonlinear potential flow code. We investigate the rapid spectral evolution due to third-order interactions and assess the effects of steepness, finite depth, directional spreading and the tail of the spectrum. We also consider the implications of third-order interactions for the shape, lifetime and kinematics of the wave groups, as well as the implications for wave loads acting on a column. Our simulations indicate that the directional energy transfers of a narrow-banded wave group initially resemble the Phillips resonance loop but agreement deteriorates as the wave group becomes more broad-banded. We also observe that the localised nonlinear features of an individual steep wave event may depend on the spectral equilibrium of the underlying sea state.

\section{Details of the numerical simulations}

We perform numerical simulations with the fully-nonlinear potential flow code OceanWave3D, described in Engsig-Karup et al. (2009). Time marching of the initial conditions has been performed both excluding and including the nonlinear terms in the freesurface boundary conditions. The linear and nonlinear simulations begin with identical amplitudes and phases for the wave components, highlighting the influence of wave-wave interactions. We use the method of phase separation (Fitzgerald et al. (2014)) to remove the bound harmonics from the nonlinear simulations, isolating the free wave components. 


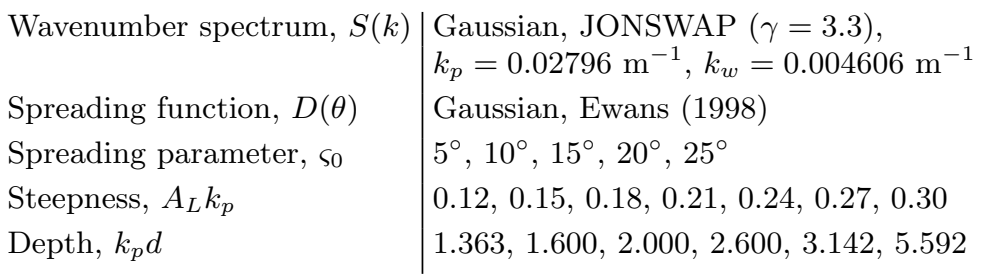

TABle 1. Parameter space of initial conditions.

\subsection{Initial conditions}

We prescribe the initial conditions in terms of surface elevation, $\eta(x, y, t)$, and velocity potential at the free surface, $\tilde{\phi}(x, y, t)$, calculated 15 characteristic wave periods before the time of linear focus. Time is thus defined relative to the linear focus time $\left(t / T_{0}=0\right)$, and the free-surface quantities time marched for 30 wave periods, terminating the simulation 15 wave periods after the linear focus time. Typical of the North Sea, the initial peak of the wavenumber spectrum for all simulations is located at $k_{p}=0.02796 \mathrm{~m}^{-1}$, which corresponds to a characteristic wavelength $\left(\lambda_{0}\right)$ of $225 \mathrm{~m}$ and characteristic wave period $\left(T_{0}\right)$ of $12.0-12.8 \mathrm{~s}$ for depths in the range of $48.7-200 \mathrm{~m}$.

The variance density spectrum $F(k, \theta)$ of the initial conditions has been defined as the product of a wavenumber magnitude spectrum $S(k)$ and a spreading function $D(\theta)$. Two types of wavenumber magnitude spectra are considered in this study, a Gaussian spectrum:

$$
S(k)=S_{0} \exp \left(\frac{-\left(k-k_{p}\right)^{2}}{2 k_{w}^{2}}\right),
$$

and a JONSWAP spectrum with $\gamma=3.3$ (Hasselmann et al. (1973)). The coefficient $S_{0}$ of (2.1) is taken to be unity since the focused amplitude of the wave event is determined by $A_{L}$ in (2.3). The JONSWAP spectrum represents a realistic spectrum for fetchlimited seas. Here, $k_{p}$ denotes the wavenumber corresponding to the initial peak of the wavenumber spectrum and $k_{w}$ represents the bandwidth of the Gaussian spectra. A value of $k_{p}=0.02796 \mathrm{~m}^{-1}$ has been used for both the Gaussian and JONSWAP spectra and a bandwidth of $k_{w}=0.004606 \mathrm{~m}^{-1}$ has been used for all the Gaussian cases. The Gaussian spectra have been calculated from three bandwidths below the spectral peak $\left(k=k_{p}-3 k_{w}\right)$ to three bandwidths above the spectral peak $\left(k=k_{p}+3 k_{w}\right)$ and the JONSWAP spectrum has been calculated up to $k / k_{p}=10$ based on the resolution of the grid in the OceanWave3D simulations. Note that the Gaussian wavenumber magnitude spectrum used in the study closely approximates the shape of the spectral peak for a JONSWAP spectrum with $\gamma=3.3$.

Two different spreading functions have been implemented in this study: a frequencyindependent Gaussian spreading function:

$$
D(\theta)=\frac{1}{\varsigma_{0} \sqrt{2 \pi}} \exp \left(\frac{-\theta^{2}}{2 \varsigma_{0}^{2}}\right),
$$

and the frequency-dependent spreading function of Ewans (1998). The Gaussian spreading function includes an initial spreading parameter $\left(\varsigma_{0}\right)$ listed in table 1 for the various test cases. The Ewans (1998) spreading function has been selected to account for the oblique angle of high-wavenumber components in the tail of the spectrum frequently 
observed in the field measurements of fetch-limited seas as described by Banner \& Young (1994), Hwang et al. (2000), Leckler et al. (2015) and Peureux et al. (2018).

Quasi-determinism (QD) theory, based on Boccotti (1983) and Lindgren (1970) (see Boccotti (2000) for a comprehensive review), indicates that the average shape of a an extreme event in a random, linear Gaussian field is the scaled auto-correlation function, used in this study to calculate the initial conditions of the extreme wave groups. The wave field is, thus, spatially inhomogeneous, and the linear surface elevation of the wave group, $\eta(x, y, t)$, is given by:

$$
\eta(x, y, t)=A_{L} \frac{\sum_{i, j} F\left(k_{i}, \theta_{j}\right) \cos \left(k_{i} \cos \theta_{j} x+k_{i} \sin \theta_{j} y-\omega_{i} t+\varphi_{0}\right)}{\sum_{i, j} F\left(k_{i}, \theta_{j}\right)} .
$$

Here, $k_{i}$ is the magnitude of the wavenumber for each component, $\theta_{i}$ is the direction of propagation, $A_{L}$ is the linear amplitude of the wave group at focus $\left(t / T_{0}=0\right)$ and the angular frequency of each component $\left(\omega_{i}\right)$ is calculated from the arbitrary-depth linear dispersion relationship, $\omega_{i}=\sqrt{g k_{i} \tanh \left(k_{i} d\right)}$. Table 1 lists the linear steepnesses of the simulated wave groups as well as the dimensionless depths, both based on the initial peak of the wavenumber spectrum $k_{p}$. The phase offset $\left(\varphi_{0}\right)$ is required for the four-phase separation technique (Fitzgerald et al. 2014) used to remove bound harmonics from the wave spectrum; we have performed the simulations with phase offsets of $0^{\circ}, 90^{\circ}, 180^{\circ}$, $270^{\circ}$ for every case, which allows the free harmonics to be approximately separated from the bound harmonics. We apply exact second-order corrections to the initial conditions based on the formulation of Dalzell (1999) together with an approximate form of thirdorder correction based on Barratt et al. (2020). Note that we evaluate the second-order terms of Dalzell (1999) at the mean water level but we evaluate the linear terms at the free surface to minimise the formation of second-order error waves.

\subsection{Code description and simulation fidelity}

Ocean Wave3D discretizes the potential flow equations for surface gravity waves in a three-dimensional spatial domain using Cartesian coordinates $(x, y, z)$. The code is based upon an Eulerian frame of reference and cannot capture overturning waves. A nonconformal transform is used to map the solution to a time-invariant domain comprised of structured rectilinear grids:

$$
\sigma \equiv \frac{z+d(x, y)}{\eta(x, y, t)+d(x, y)}
$$

and uniform depth, $d(x, y)=d$, in this study. Classic fourth-order Runge-Kutta (RK4) time marching combined with finite differencing of the spatial derivatives yields a linear system of equations, solved with the Generalised Minimum Residual (GMRES) algorithm. Left preconditioning is performed once every iteration with multi-grid GaussSeidel relaxation. The multi-grid scheme is based on direct coarse grid approximation and follows a 'V-cycle', descending from the finest grid resolution down to the coarsest grid resolution before ascending again from the coarsest grid resolution to the finest grid resolution, with one pre-smoothing and one post-smoothing step. We apply a relative tolerance of $10^{-6}$ for convergence of the GMRES algorithm at each time step.

The grid-convergence study of Barratt et al. (2020), based on grid halving in all three spatial dimensions, informs our selection of the grid resolution. The size of the numerical domain is listed in table 2 , indicating the length $(L)$ in the $x$-direction, width $(W)$ in the $y$-direction, and depth $(d)$ in the vertical, $z$-direction. The length and width of the domain are fixed for all simulations. However, we vary the depth of the domain. The 


\begin{tabular}{l|l}
$\begin{array}{l}\text { Characteristic time } \\
\text { and length scales }\end{array}$ & $\begin{array}{l}\text { Wavelength }\left(\lambda_{0}\right) 225 \mathrm{~m} \\
\text { Wave period* }\left(T_{0}\right) 12.0-12.8 \mathrm{~s}\end{array}$ \\
\hline Numerical domain & $\begin{array}{l}\text { Length }(L) 7680 \mathrm{~m} \\
\text { Width }^{\dagger}(W) 2560 \mathrm{~m} \\
\text { Depth }(d) 48.7-200 \mathrm{~m} \\
\dagger\end{array}$ \\
\hline Out from symmetry plane at $y=0$
\end{tabular}

TABLE 2. Numerical details of OceanWave3D simulations.

horizontal grid distribution $(\Delta x, \Delta y)$ is uniform throughout the domain. We utilise a symmetry plane along the centreline of the wave group $(y=0)$. Thus, the width of the domain listed in table 2 only represents half of the effective domain width. The position of the symmetry plane results in asymmetric stencils along the center of the wave group. However, by performing simulations with and without a symmetry plane, Barratt et al. (2020) confirmed that the additional numerical diffusion due to the asymmetric stencils is negligible. We use powers of two for the number of grids $\left(N_{x}, N_{y}, N_{z}\right)$ in all three spatial dimensions, to ensure the maximum number of multi-grid levels $\left(N_{M G}\right)$ for the preconditioning scheme. The number of grids in the horizontal directions $\left(N_{x}, N_{y}\right)$ has been doubled for the JONSWAP cases, compared with the Gaussian cases, to better resolve the smaller-wavelength components in the tail of the JONSWAP spectrum.

We use eighth-order finite differencing, confirmed by Barratt et al. (2020) to exhibit a grid-independent iteration count for simulations similar to those of this study. Centraldifferencing schemes have been used, except at the boundaries, and the order of finite differencing is consistent throughout. We include a single layer of ghost nodes, outside the domain of interest, for all surfaces except the free surface. The presence of ghost nodes at the bottom boundary allows both continuity and the Neumann boundary condition to be simultaneously satisfied while improving the numerical stability of the preconditioning scheme, even for anisotropic grid distributions in the vertical direction. Thus, we use the symmetric half of a Chebyshev-Gauss-Lobatto (CGL) distribution in the vertical direction: $\sigma_{j}=\sin \left((\pi[j-1]) /\left(2\left[N_{z}-1\right]\right)\right)$, where $j$ denotes the index of the grid point with $j=1$ at the bottom and $j=N_{z}$ at the free surface. The time step size, based on the Courant-Friedrichs-Lewy (CFL) condition, influences diffusion error but not dispersion 
error. Thus, we use the same time step for the JONSWAP and Gaussian cases based upon the permissible levels of diffusivity, resulting in CFL values of 1.0 and 0.5 respectively.

\subsection{Spectral parameters}

We analyse the spectral evolution of the various simulations performed in this study in terms of moments of the wavenumber-amplitude spectrum as well as spreading bandwidth. We also consider the wave action density spectrum to determine the timescales of energy transfer between wave components. The zeroth moment of the discrete wavenumber-amplitude spectrum $\left(\mathcal{M}_{0}\right)$ :

$$
\mathcal{M}_{0}=\sum_{i}\left|\hat{\eta}\left(k_{i}, \theta_{i}\right)\right|
$$

represents a summation of amplitudes for the Fourier components of surface elevation in the discrete wavenumber spectrum, $\hat{\eta}\left(k_{i}, \theta_{i}\right)$, and thus represents the amplitude of the wave which would be formed by perfect constructive interference between all wave components. For linear evolution, $\mathcal{M}_{0}$ in (2.5) equals $A_{L}$ as defined in (2.3) for the entirety of the simulation. The zeroth spectral moment $\mathcal{M}_{0}$ can be expected to increase as energy transfers in a narrow-banded spectrum lead to broadening of the spectrum. We use the first moment of the wavenumber-amplitude spectrum

$$
\mathcal{M}_{1}=\sum_{i} k_{i}\left|\hat{\eta}\left(k_{i}, \theta_{i}\right)\right|
$$

normalised by the zeroth spectral moment $\left(\mathcal{M}_{0}\right)$ to define a mean wavenumber $(\mathcal{K})$ :

$$
\mathcal{K}=\mathcal{M}_{1} / \mathcal{M}_{0}
$$

The mean wavenumber $\mathcal{K}$ increases if high-wavenumber components grow in amplitude while low-wavenumber components diminish in amplitude. Thus, $\mathcal{K}$ increases if energy transfer from low-wavenumber components to high-wavenumber components prevails and decreases in the opposite scenario. We also consider the root-mean-square (RMS) spreading $(\varsigma)$ :

$$
\varsigma=\sqrt{\frac{\sum_{i} \theta_{i}{ }^{2}\left|\hat{\eta}\left(k_{i}, \theta_{i}\right)\right|}{\mathcal{M}_{0}}},
$$

which quantifies the bandwidth of directional spreading. Note that the initial value of (2.8) matches the parameter $\varsigma_{0}$ in (2.2) if the frequency-independent Gaussian spreading function is used. The spectral parameters defined in (2.7) and (2.8) have been evaluated for both the Gaussian and JONSWAP cases based on wavenumber components in the range: $0<k_{x} / k_{p} \leqslant 2$ and $-2 \leqslant k_{y} / k_{p} \leqslant 2$. We restrict the calculation to the vicinity of the spectral peak to assess the Gaussian spectrum as a model for the peak of a JONSWAP spectrum. Thus, we do not include the full tail of the JONSWAP spectrum in the calculation of the spectral parameters. However, we note that the considered range of wavenumbers accounts for $96-98 \%$ of the total energy for the JONSWAP cases, calculated at every time step for each simulation.

The total wave action of a wave group is a conserved quantity (see Komen et al. (1994)), and the spectrum of wave action density has been calculated from the amplitude spectrum to quantify the component growth rates. The energy density spectrum $E\left(k_{i}, \theta_{i}\right)$, associated with the amplitude spectrum of surface elevation in wavenumber space, has been calculated based upon the amplitude of each Fourier component, $a_{i}$, and the discrete 
wave number intervals $\left(\Delta k_{x}, \Delta k_{y}\right)$ together with the density of water $(\rho)$ :

$$
E\left(k_{i}\right)=\frac{\frac{1}{2} \rho g a_{i}^{2}}{\Delta k_{x} \Delta k_{y}} .
$$

Using the linear dispersion relation, the wave action density spectrum is given by:

$$
A\left(k_{i}\right)=\frac{E\left(k_{i}\right)}{\omega_{i}}
$$

In this study, we consider the growth rate of specific wave components. For context, Stiassnie (2001) notes that the growth rate associated with the Hasselmann equation is:

$$
\left[\partial \mathcal{A}\left(k_{i}, \theta_{i}\right) / \partial t\right] T_{0} / \mathcal{A}\left(k_{i}, \theta_{i}\right)=O\left(\epsilon^{4} \omega_{0} T_{0}\right),
$$

where $\omega_{0}$ represents the characteristic wave period, and the growth rate associated with the Zakharov equation is:

$$
\left[\partial \mathcal{A}\left(k_{i}, \theta_{i}\right) / \partial t\right] T_{0} / \mathcal{A}\left(k_{i}, \theta_{i}\right)=O\left(\epsilon^{2} \omega_{0} T_{0}\right) .
$$

\section{Results and discussion}

We begin our analysis with a base case, followed by an investigation of steepness, finite depth, directional spreading and the high-wavenumber tail of the spectrum.

\subsection{Directional energy transfers due to third-order interactions}

The selected base case features a Gaussian wavenumber spectrum with a Gaussian spreading function and a spreading parameter $(\varsigma)$ of $15^{\circ}$, denoted as case 'GG15'. We prescribe a linear steepness $\left(A_{L} k_{p}\right)$ of 0.3 for the base case at the linear focus time $\left(t / T_{0}=0\right)$, from $(2.3)$, together with a dimensionless depth $\left(k_{p} d\right)$ of 3.142 . Results are shown for the linear version of the base case, in which the nonlinear terms of the free surface boundary conditions are neglected, and the fully nonlinear version of the base case which includes all terms in the free-surface boundary conditions. Figure 1 shows the surface elevation corresponding to the nonlinear version of the base case, including the wing waves (demarcated with $\mathcal{W}$ ) which form as a result of directional energy transfers.

The steepness of the wave envelope over time $\left(A(t) k_{p}\right)$ is shown in figure $2(\mathrm{a})$ for both the linear and nonlinear version of the base case. As expected, the linear envelope is symmetric about the focal time. However, the nonlinear envelope exhibits asymmetry about $t / T_{p}=0$ due to nonlinear wave-wave interactions which modify the amplitude and dispersion of the wave components, extending the life span of this focused wave event. Nonlinear interactions are known to alter the phase speed of the participating wave components, which can counteract dispersion and preserve the form of a focused wave event - the competing effects of nonlinearity and dispersion is a central idea to the Benjamin-Feir index (see Janssen (2003)). Figure 2(b) shows the zeroth spectral moment of the amplitude spectrum $\left(\mathcal{M}_{0}\right)$, normalised by the linear focus amplitude $\left(A_{L}\right)$. For linear evolution, the ratio equals unity at all times. However, for nonlinear evolution the zeroth moment can be seen to increase during the focusing of the wave group reaching a maximum value of $\mathcal{M}_{0} / A_{L}=1.7$ at $t / T_{0}=8.0$ in figure $2(\mathrm{~b})$. Although the zeroth moment increases continuously during the wave focusing event, the amplitude of the nonlinear event at focus is smaller than the amplitude of the linear event due to modifications in phase speed. For the linear simulation, the phase of each component has been selected to ensure perfect constructive interference at $t / T_{0}=0$. For the nonlinear simulation, nonlinear dispersion prevents perfect constructive interference at focus. Thus, 


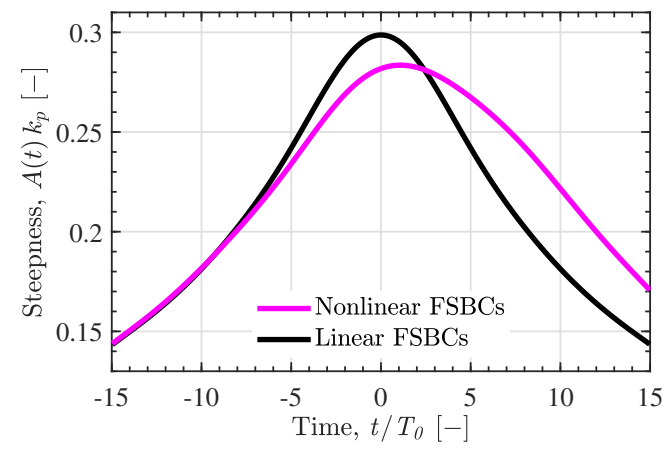

(a)

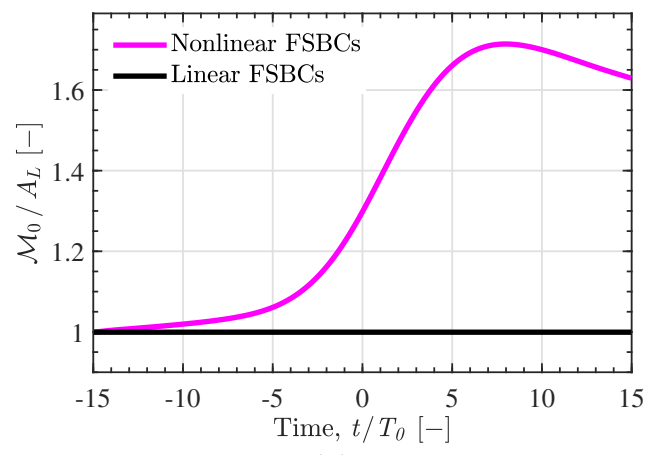

(b)

FiguRE 2. Comparison for case GG15 with linear and nonlinear free-surface boundary conditions (FSBCs), a steepness of $A_{L} k_{p}=0.3$ and a dimensionless depth of $k_{p} d=3.142:(a)$ Steepness of the wave envelope over time. The amplitude $A(t)$ is based on the maximum elevation of the wave envelope at time $t$. The linear case is represented by the black line ( - ) and the nonlinear case is represented by the magenta line ( $)$. (b) The zeroth moment of the wavenumber-amplitude spectrum $\left(\mathcal{M}_{0}\right)$ normalised by the linear focus amplitude $\left(A_{L}\right)$. The dimensionless ratio is plotted over time for the nonlinear case ( $)$ ) and the linear case $(\mathbf{C})$. For the linear case, the ratio $\mathcal{M}_{0} / A_{L}$ equals unity at all instances in time.

the steepness of the focused event is reduced, despite the increase in zeroth moment shown in figure 2(b). Although the spectral evolution of the nonlinear event does not increase the amplitude of the wave envelope in this case, the wave-wave interactions significantly alter the shape of the wave group and the magnitude of the wave kinematics, discussed in section 3.2. Adjusting the initial phase of the wave components could evidently result in a nonlinear event which is locally larger than the linear version of the event.

For the base case, we also consider the evolution of the wave spectrum due to nonlinear wave-wave interactions, calculated with a Discrete Fourier Transform (DFT) of the wave group surface elevation. Figure 3 depicts contour plots of the amplitude spectrum in wavenumber space for: the initial condition, $t / T_{0}=-15$; the time of nonlinear focus, $t / T_{0}=1.3$; and the end of the simulation, $t / T_{0}=15$. Segments of the Phillips 'figureof-eight' resonance loop are also shown. The initial condition in figure 3(a) is narrowbanded and remains narrow-banded during the early stages of focusing due to the low steepness of the initially dispersed wave group. However, evolution of the amplitude spectrum occurs as the wave group steepens and approaches focus, see figure 2 . In particular, energy transfer to high-wavenumber components is evident in figure 3(b), with a directional bias qualitatively consistent with the Phillips resonance loop, at angles of $\pm 35.26^{\circ}$ to the spectral peak, accompanied by unidirectional energy transfers along the $k_{x}$-axis. A low-wavenumber sidelobe can be seen at nonlinear focus in figure $3(\mathrm{~b})$, although the footprint is considerably smaller than the high-wavenumber sidelobe. The spectral changes observed in figure 3(b) are, thus, reminiscent of the Class I instability band for a regular wave train of finite amplitude, described by McLean (1982b), and we attribute the spectral changes to 'quasi-degenerate' interactions. A degenerate quartet is comprised of only three unique wave components (since one component participates twice, $\boldsymbol{k}_{1}=\boldsymbol{k}_{2}$ ) and forms the basis of stability analyses for a regular/monochromatic wave, assuming that $\boldsymbol{k}_{1}$ and $\boldsymbol{k}_{2}$ both equate to the characteristic wavenumber $\boldsymbol{k}_{0}$ of the regular wave. Phillips (1960) showed that the interacting components of a degenerate quartet form the 'figure-of-eight' resonance loop, and the eigenvalue stability analysis of McLean (1982b) indicates that the instability band of a finite-amplitude regular wave 

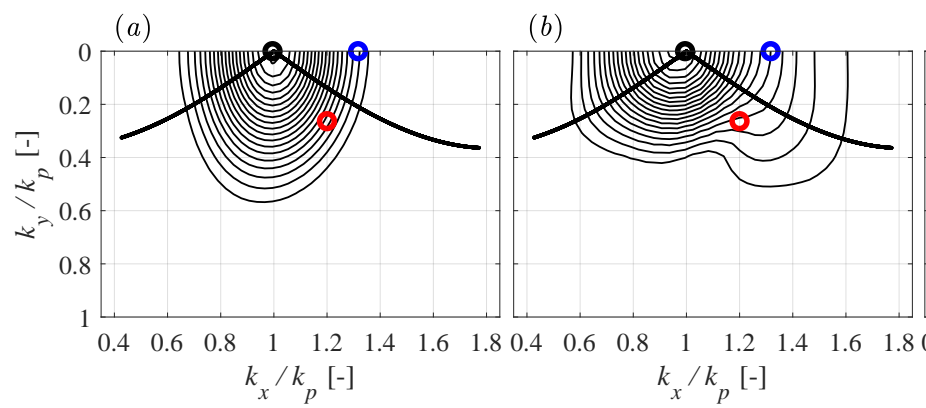

$(c)$

Figure 3. Discrete amplitude spectra of surface elevation corresponding to case GG15 with a steepness of $A_{L} k_{p}=0.3$ and a dimensionless depth of $k_{p} d=3.142$ : (a) initial condition, $t / T_{0}=-15$; (b) nonlinear focus, $t / T_{0}=1.3$; (c) post-focus, $t / T_{0}=15$. Contour levels $(-)$ are evenly distributed between $0.01 \mathrm{~m}$ and $0.105 \mathrm{~m}$ in intervals of $0.005 \mathrm{~m}$. The thick black line (-) represents a segment of the Phillips 'figure-of-eight' resonance loop and the open circles specify particular wavenumber components for further analysis: $\mathcal{Q}(\mathbf{0}) k_{x} / k_{p}=0.995$, $k_{y} / k_{p}=0.000 ; \mathcal{R}(\mathbf{O}) k_{x} / k_{p}=1.200, k_{y} / k_{p}=0.263 ; \mathcal{S}(\mathbf{0}) k_{x} / k_{p}=1.317, k_{y} / k_{p}=0.000$.

includes components on the $k_{x}$-axis if the water is deep. In this study, we consider narrowbanded wave spectra which form focused wave groups. The concentration of energy around the spectral peak means that we anticipate interactions in which $\boldsymbol{k}_{1}$ and $\boldsymbol{k}_{2}$ resemble the characteristic wavenumber $\boldsymbol{k}_{0}$ (wavenumber of the initial spectral peak) but may not strictly equate. Thus, we expect $\boldsymbol{k}_{1} \approx \boldsymbol{k}_{2} \approx \boldsymbol{k}_{0}$ for our narrow-banded wave groups, rather than $\boldsymbol{k}_{1}=\boldsymbol{k}_{2}=\boldsymbol{k}_{0}$ as expected for a regular/monochromatic wave. Interactions of the form $\boldsymbol{k}_{1} \approx \boldsymbol{k}_{2}$ are not strictly degenerate. Thus, we use the term 'quasi-degenerate' to describe quartet interactions with $\boldsymbol{k}_{1} \approx \boldsymbol{k}_{2}$. Despite the resemblance of the directional energy transfers figure 3(b) to the Phillips resonance loop, it seems improbable that all the directional energy transfers in figure 3(b) are the result of exactly degenerate quartets, due to the strict definition. Thus, we attribute the directional energy transfers evident in 3(b) to quasi-degenerate interactions since the definition of quasidegenerate interactions is more relaxed and encompasses a large number of interactions with resonance loops that resemble the classic Phillips resonance loop. After nonlinear focus, figure 3(c), the amplitude spectrum continues to broaden with directional energy transfer to high-wavenumber components not encompassed by the Phillips resonance loop. Divergence from the figure-of-eight is likely due to the degenerate nature of the resonance loop. Broadening of the spectrum during focusing, thus, appears to facilitate non-degenerate interactions $\left(\boldsymbol{k}_{1} \neq \boldsymbol{k}_{2}\right)$ after focus, resulting in high-angle oblique energy transfers along a $\pm 55^{\circ}$ angle. Notably, there are spectral changes which arise during focus that do not reverse after focus indicating permanent changes to the spectrum. We note that suppressed linear dispersion has been observed by Steer et al. (2019) for modulations which propagate at $\pm 35.26^{\circ}$ to the wave group, which can increase the lifetime of a focused wave event. Thus, the $\pm 35.26^{\circ}$ energy transfers apparent in figure 3(b) may contribute to the lifetime extension depicted in figure 2(a). The formation of a high-wavenumber sidelobe has also been associated by Adcock \& Taylor (2016) with contraction of the wave group in the direction of propagation. The amplitude spectrum at nonlinear focus also exhibits a contraction of the spectrum along the $k_{y}$-direction, which implies lateral expansion of the wave group and a focused wave event which is less directionally spread than the initial wave spectrum.

Three specific components in the wavenumber spectrum $(\mathcal{Q}, \mathcal{R}, \mathcal{S})$ have been selected for further analysis of the growth rate - the components are shown in figure 3 and listed 


\begin{tabular}{c|c|c|c} 
& $\mathcal{Q}$ & $\mathcal{R}$ & $\mathcal{S}$ \\
\hline$k_{x} / k_{p}$ & 0.995 & 1.200 & 1.317 \\
$k_{y} / k_{p}$ & 0.000 & 0.263 & 0.000 \\
$|\boldsymbol{k}| / k_{p}$ & 0.995 & 1.228 & 1.317
\end{tabular}

TABLE 3. Wavenumber components selected for growth analysis shown in figure 3.

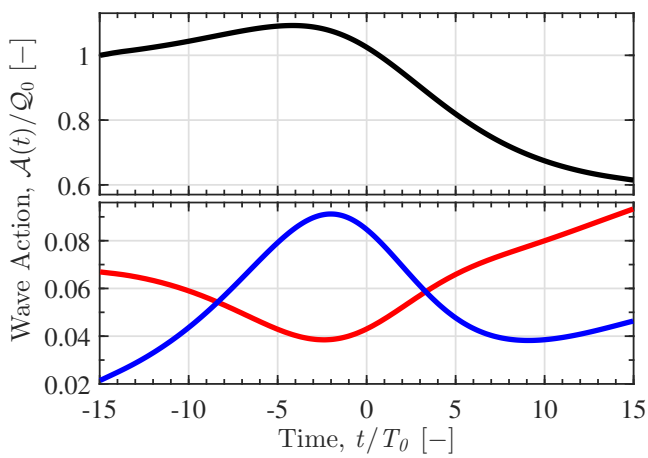

(a)

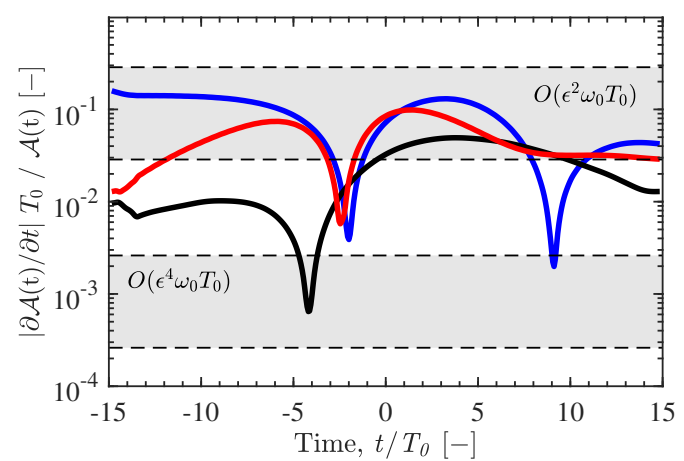

(b)

FiguRE 4. Component-wise analysis of the wave action density spectrum for case GG15 with a steepness of $A_{L} k_{p}=0.3$ and a dimensionless depth of $k_{p} d=3.142$ : (a) the wave action density of each component over time, normalised by the initial wave action density of the spectral peak $\left(\mathcal{Q}_{0}\right)$; (b) the nondimensional growth rate of each component over time, plotted on a logarithmic ordinate. The components correspond to those indicated in figure 3: $\mathcal{Q}(-) k_{x} / k_{p}=0.995$, $k_{y} / k_{p}=0.000 ; \mathcal{R}(-) k_{x} / k_{p}=1.200, k_{y} / k_{p}=0.263 ; \mathcal{S}(\longrightarrow) k_{x} / k_{p}=1.317, k_{y} / k_{p}=0.000$. The dynamic growth rate $O\left(\epsilon^{2} \omega_{0} T_{0}\right)$ and the kinetic growth rate $O\left(\epsilon^{4} \omega_{0} T_{0}\right)$ are indicated by bounded (- - ) grey bands. The value of $\epsilon$ equates to $A_{L} k_{p} / \pi$ in the growth rate calculations.

in table 3. Component $\mathcal{Q}$ coincides with the initial peak of the wavenumber spectrum, component $\mathcal{S}$ denotes a component on the $k_{x}$-axis, in the instability band of a finiteamplitude regular wave train, and component $\mathcal{R}$ coincides with the local maximum of the oblique ridge at $\pm 55^{\circ}$ formed by non-degenerate interactions. Figure $4(\mathrm{a})$ shows the wave action density of the three components. Before focus, the wave action density of component $\mathcal{Q}$ grows, indicating energy transfer to the spectral peak, while the wave action density of component $\mathcal{R}$ declines, due to contraction of the spectrum in the $k_{y^{-}}$ direction, and component $\mathcal{S}$ grows, due to energy transfers to higher wavenumbers. Before nonlinear focus occurs at $t / T_{0}=1.3$, the wave action density of component $\mathcal{Q}$ begins to decline, signalling the onset of a downshift in the spectral peak, and component $\mathcal{R}$ begins to grow, continuing to grow for the remainder of the simulation. Component $\mathcal{S}$ reaches a maximum in wave action density before nonlinear focus and thereafter begins to decline, which is likely a result of spectral broadening inhibiting quasi-degenerate interactions. Previous work by Longuet-Higgins (1978), McLean (1982b), Francius \& Kharif (2003) and Fedele (2014) has also demonstrated the suppression of unidirectional instabilities at high wave steepnesses, which may also constrain the growth in wave action density for component $\mathcal{S}$ during focus. The corresponding dimensionless growth rates for the three components $(\mathcal{Q}, \mathcal{R}, \mathcal{S})$ are shown in figure $4(\mathrm{~b})$ in terms of wave action density. For context, the dynamic timescale associated with near-resonance and the Zakharov 
equation, $O\left(\epsilon^{2} \omega_{0} T_{0}\right)$, is shown together with the kinetic timescale associated with exact resonance and the Hasselmann equation, $O\left(\epsilon^{4} \omega_{0} T_{0}\right)$.

Similar results to figure 3 have been observed by Adcock \& Taylor (2016) using the MNLS equation of Trulsen \& Dysthe (1996). Thus, these spectral changes can be attributed to resonant third-order interactions, since the MNLS equation is only capable of resolving third-order/four-wave interactions, as demonstrated by Stiassnie (1984). Furthermore, the HOS simulations of Gibbs \& Taylor (2005) were performed with a truncated form of Dirichlet-Neumann operator and yielded results similar to figure 3 ; the authors indicated that a comparison of third-order and fifth-order simulations produced no significant variations. Thus, the fourth-order Class II instabilities observed by McLean (1982b) do not appear to be significant for the simulated wave groups in this study. Although the present study is focused on isolated wave groups, the oblique energy transfers and consequent spectral evolution shown in figure 3 qualitatively resemble previous random sea results (e.g., Dysthe et al. (2003), Socquet-Juglard et al. (2005), Toffoli et al. (2010), Xiao et al. (2013), Simanesew et al. (2016) and Simanesew et al. (2018)). Toffoli et al. (2010), for example, used Higher Order Spectral (HOS) simulations to demonstrate that a random wave field initialised with frequency-independent spreading develops frequency-dependent spreading resembling figure 3 . The simulations of Toffoli et al. (2010) did not include external forcing, such as wind and breaking, and the spectral evolution can thus be attributed to nonlinear wave-wave interactions. Simanesew et al. (2016) performed laboratory experiments and Modified Nonlinear Schrödinger (MNLS) simulations initialised with frequency-independent spreading and also observed the development of frequency-dependent spreading. Dynamic nonlinear contributions to the development of frequency-dependent spreading, above and below the spectral peak, were identified, consistent with our observations. Simanesew et al. (2018) investigated wave spectra based on field measurements from the North Sea and MNLS simulations, using different data-adaptive methods and indicate that frequency-dependent spreading can be a numerical artefact of 'peak-splitting' by maximum entropy methods. However, Simanesew et al. (2018) conclude that the development of frequency-dependent spreading in the spectral tail does also result from nonlinear wave-wave interactions, consistent with our results. Thus, the local spectral evolution surrounding steep wave events, featuring oblique energy transfers away from the spectral peak, may contribute to the global evolution of the underlying sea-state. The development of frequency-dependent spreading does, however, occur slower for the background sea-state of a random sea requiring hundreds of wave periods to fully develop. In contrast, significant frequency-dependent spreading develops within 30 wave periods in the present study of steep wave groups.

\subsection{Implications of spectral changes for kinematics and loads}

The spectral changes observed in figure 3 are relevant to the kinematics of the wave group. The wave loads acting upon a vertical cylinder can be calculated with the Morison equation, see for example Paulsen et al. (2014), yielding the horizontal force:

$$
F=\int_{-d}^{\eta} \frac{1}{2} C_{D} \rho A u(z)|u(z)| d z+\int_{-d}^{\eta} C_{M} \rho V \frac{D u(z)}{D t} d z,
$$

featuring a drag term with a quadratic dependency on the horizontal velocity, $u|u|$, and an inertial term proportional to the Lagrangian acceleration of the horizontal velocity, $D u / D t$, defined in terms of the material derivative. Here, $u(z)$ represents the characteristic horizontal velocity which may vary in the vertical, $z$-direction. Constants $C_{D}$ and $C_{M}$ depend on geometry and Reynolds number. The frontal area of the body $(A)$ as well as the volume $(V)$ are required for the calculation, together with the density of 


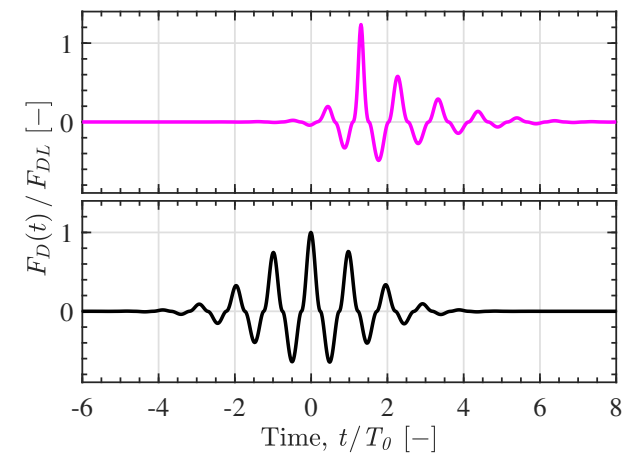

(a)

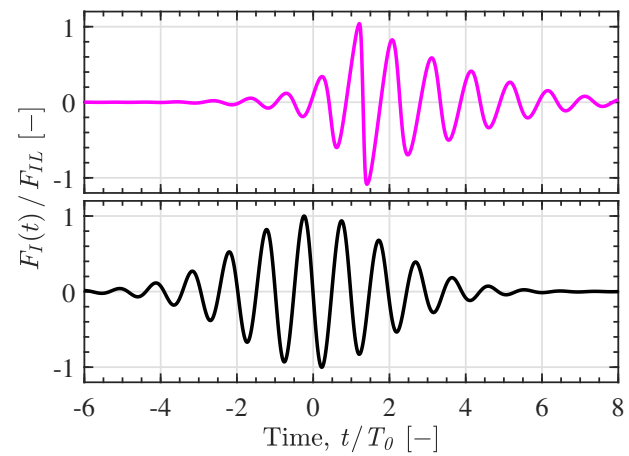

(b)

FiguRE 5. Time-history of force calculated from the terms of the Morison equation for case GG15 with a steepness of $A_{L} k_{p}=0.3$ and a dimensionless depth of $k_{p} d=3.142$ : (a) drag force corresponding to the $u|u|$ term of the Morison equation - the force has been normalised by the maximum drag force of the linear case $F_{D L} ;(\mathrm{b})$ inertial force corresponding to the $\partial u / \partial t$ term of the Morison equation - the force has been normalised by the maximum inertial force of the linear case $F_{I L}$. The linear case is depicted by the black line ( - ) and the nonlinear case is depicted by the magenta line ( $(-)$. The time-history of force is calculated at the spatial location where focus of the wave group occurs: $x / \lambda_{0}=0, y / \lambda_{0}=0$ for the linear case and $x / \lambda_{0}=1.535, y / \lambda_{0}=0$ for the nonlinear case, by integrating from the bottom of the domain $(z=-d)$ to the surface elevation $(z=\eta)$.

the fluid $(\rho)$. Energy transfers to higher wavenumbers augments both the velocities and accelerations of the wave field, increasing both the drag and inertial loads as estimated by the Morison equation. Furthermore, the reduction in directional spreading shown in 3(b), which occurs during focusing, increases the in-line velocity component and thus increases the horizontal velocity component used in (3.1). We have calculated (3.1) for both the linear and nonlinear versions of the base case. A time history of the force, at the spatial location where focus occurs, is plotted for both the linear and nonlinear cases - the drag term is shown in figure 5(a) and the inertial term is shown in figure 5 (b). Separate axes are used in figure 5 for the linear and nonlinear cases for clarity. The nonlinear case exhibits higher drag and inertial forces at focus than the linear case as a result of energy transfers to higher wavenumbers and the reduced spreading of the nonlinear focused event. The maximum drag and inertial forces are, respectively, $24 \%$ and $4 \%$ higher for the nonlinear case, compared with the linear case. The shape of the linear and nonlinear focused events is shown in figure 6 with contours of the in-line velocity component $(u)$, aligned to the $x$-direction, and normalised by the phase speed associated with the spectral peak $\left(c_{0}\right)$. The linear event depicted in figure $6(\mathrm{a})$ exhibits front-rear symmetry, and the height of the crest is comparable to the depth of the preceding and trailing troughs; a peak inline velocity of $u / c_{0}=0.294$ arises in the crest of the linear focused event. For the nonlinear event depicted in figure 6(b), front-rear asymmetry is apparent as the preceding trough is shallower than the trailing trough. The surface elevation includes bound harmonics which result in a higher crest and shallower troughs than observed for the linear event; a peak inline velocity of $u / c_{0}=0.449$ arises in the crest of the nonlinear focused event, $53 \%$ higher than the linear case. The Particle Image Velocimetry (PIV) experiments of Alberello et al. (2018) are consistent with the numerical simulation results shown in figure 6. Alberello et al. (2018) observed that unidirectional wave groups, formed by dispersive focusing, contract in the direction of propagation and exhibit asymmetric features consistent with figure 6 : a preceding trough that is shallower 


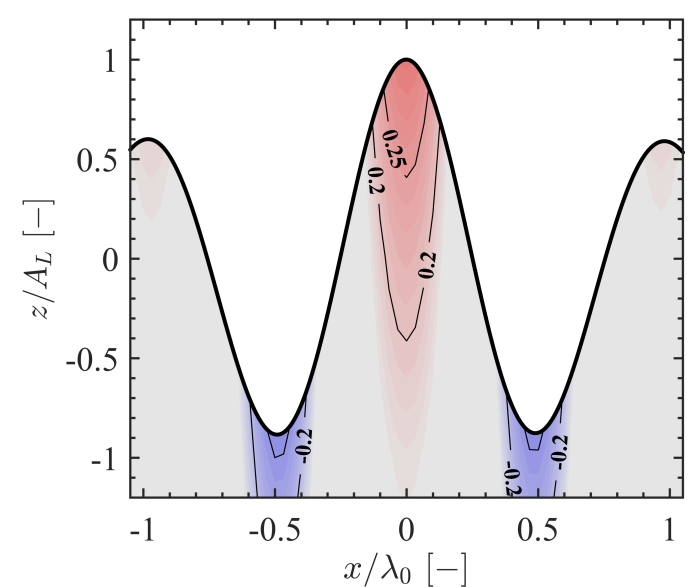

(a)

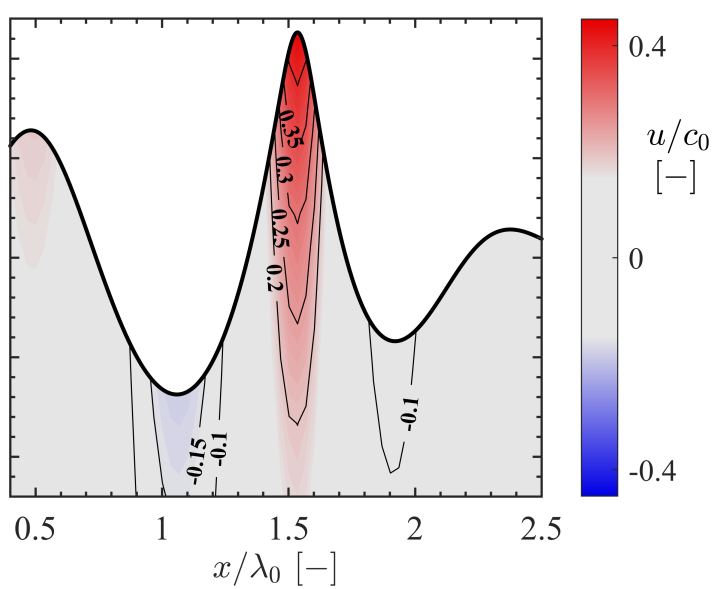

(b)

FiguRE 6. Internal kinematics at the time of focus for case GG15 with a steepness of $A_{L} k_{p}=0.3$ and a dimensionless depth of $k_{p} d=3.142$ : $(a)$ Linear evolution, focus occurs at $t / T_{0}=0 ;(b)$ Nonlinear evolution, focus occurs at $t / T_{0}=1.3$. The spatial coordinate in the direction of propagation ( $x$-direction) is shown on the horizontal axis and the vertical spatial coordinate ( $z$-direction) is shown on the vertical axis. The horizontal velocity in the direction of wave group propagation ( $u$-velocity) is shown beneath the surface elevation ( - ) of the wave group, normalised by the phase speed associated with the initial peak of the wavenumber spectrum $\left(c_{0}\right)$. Labelled contour lines of the dimensionless velocity ratio $u / c_{0}$ are shown in the figures together with a colour bar of the same parameter.

than the trailing trough and a focused crest that is sharper than both the preceding and trailing trough. The consequent kinematics measured by Alberello et al. (2018) featured concentrated high-velocity regions in the crest, similar to figure 6 , associated with the onset of breaking. Note that a variety of breaking criteria have been proposed based on crest kinematics/energy-fluxes (see, e.g., Barthelemy et al. (2018)) and neither of the focused events shown in figure 6 are expected to break (see Perlin et al. (2013) for a review of breaking criteria). In summary, the spectral evolution observed in the base case GG15 results in augmented kinematics and loads, demonstrating the significance of nonlinear interactions.

\subsection{Steepness, finite depth and directional spreading effects}

Following our analysis of the base case, we investigate the effects of steepness, finite depth and directional spreading on spectral evolution on the mean wavenumber $(\mathcal{K})$ and the spreading parameter $(\varsigma)$.

\subsubsection{Steepness}

We assess the effect of amplitude for wave groups based on a Gaussian wavenumber spectrum and a Gaussian spreading function with an initial spreading parameter $\left(\varsigma_{0}\right)$ of $15^{\circ}$ and a dimensionless depth of $k_{p} d=3.142$. The linear steepness at focus is varied between 0.12 and 0.30 in intervals of 0.03 . The mean wavenumber, defined in (2.7), and spreading parameter, defined in (2.8), are shown in figure 7 . The vertical axis of both plots is normalised by the initial value of the parameter. The evolution in mean wavenumber, shown in figure $7(\mathrm{a})$, depicts an increase during focus with a rate dependent upon steepness. The increase during focus is predominantly due to the quasidegenerate interactions, discussed in section 3.1, which result in energy transfers to higher 


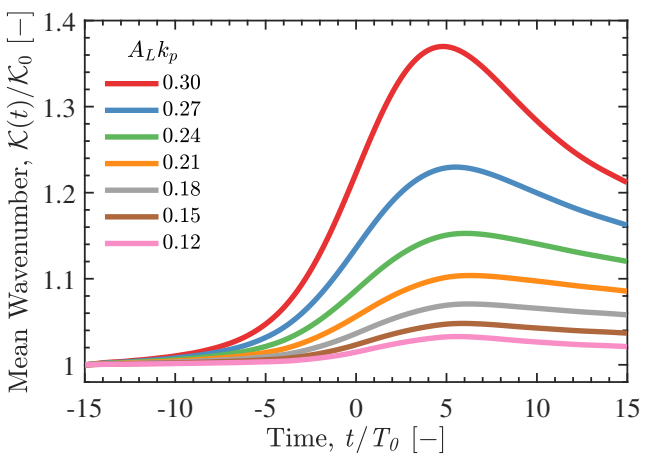

(a)

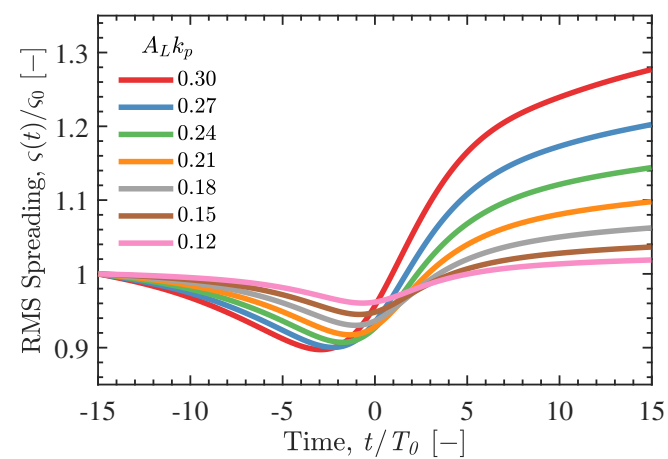

(b)

FiguRE 7. Spectral evolution over time for case GG15 with different linear steepnesses $\left(A_{L} k_{p}\right)$ and a dimensionless depth of $k_{p} d=3.142$ : (a) mean wavenumber $\mathcal{K}(t)$ normalised by the initial value $\mathcal{K}_{0}$; (b) RMS spreading parameter $\varsigma(t)$ normalised by the initial value $\varsigma_{0}$. The various steepnesses are indicated by colour: $A_{L} k_{p}=0.30, \longleftarrow ; 0.27, \longleftarrow ; 0.24, \longleftarrow ; 0.21, \square$; $0.18,=; 0.15,=$ -

wavenumbers. After focus, the mean wavenumber peaks and thereafter declines for all cases. The post-focus decline in mean wavenumber is due to the post-focus dominance of the non-degenerate interactions discussed in section 3.1-the high oblique angle components possess a smaller absolute wavenumber than those along the unidirectional $k_{x}$-axis, thereby decreasing the mean wavenumber. Figure $7(\mathrm{~b})$ shows the corresponding evolution of the spreading parameter $(\varsigma)$, which exhibits a qualitatively similar trend for all steepnesses. The spreading parameter initially declines during focusing, indicating a more unidirectional wave group resembling the 'wall of water' effect described by Gibbs \& Taylor (2005) and observed experimentally by Steer et al. (2019), followed by a rapid increase in directional spreading due to the oblique non-degenerate energy transfers shown in figure 3(c). The increase in directional spreading occurs earlier for the steeper wave groups indicating an earlier onset of the non-degenerate interactions.

\subsubsection{Finite depth}

The effect of finite depth has also been assessed for wave groups based on a Gaussian wavenumber spectrum and a Gaussian spreading function with an initial spreading parameter $\left(\varsigma_{0}\right)$ of $15^{\circ}$ and a linear steepness $\left(A_{L} k_{p}\right)$ of 0.30 , with the results shown in figure 8 . The considered range of depths spans from $k_{p} d=5.592$ to $k_{p} d=1.363$; a depth of $k_{p} d=3.142$ is frequently considered to be 'deep water', and the lower bound of $k_{p} d=1.363$ is significant as the critical depth of vanishing modulational instability for unidirectional waves (see Benjamin (1967); Whitham (1967)). However, Benney \& Roskes (1969) and McLean (1982a) showed that unstable three-dimensional perturbations remain for depths less than $k_{p} d=1.363$, consistent with our findings - we observe suppressed nonlinear evolution of the wave spectrum for a depth of $k_{p} d=1.363$ but rapid energy transfers evidently do still occur.

Figure 8 (a) depicts the evolution of the mean wavenumber, normalised by the initial value, for various finite depths. A reduction in depth suppresses the increase in mean wavenumber observed during focus but a discernible increase remains apparent for $k_{p} d=$ 1.363. We also observe sensitivity to depth between $k_{p} d$ values typically considered to represent 'deep water'. Depths of $k_{p} d=5.592$ and $k_{p} d=3.142$ result in peak $\mathcal{K}(t) / \mathcal{K}_{0}$ values of 1.44 and 1.37, respectively. Figure 9 shows the amplitude spectrum of surface elevation at the end of the simulation $\left(t / T_{0}=15\right)$ for the various finite-depth cases. The 


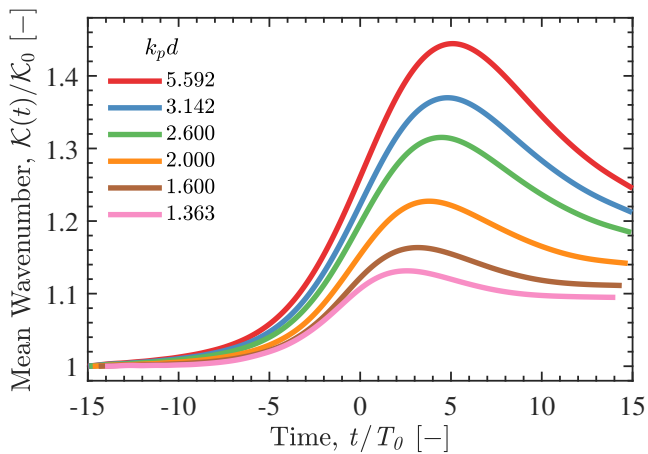

(a)

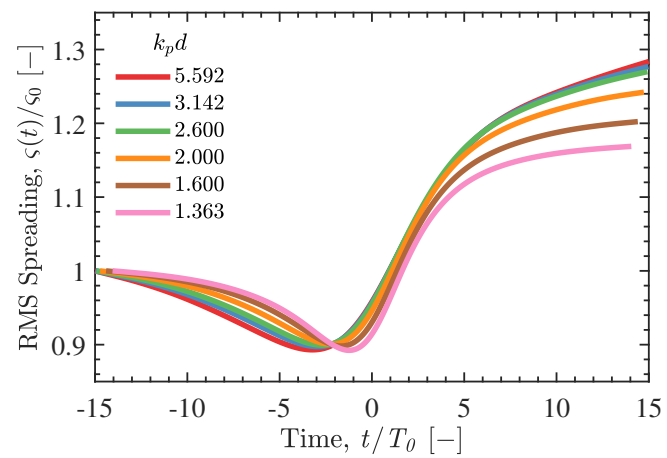

(b)

FiguRE 8. Spectral evolution over time for case GG15 with different dimensionless depths $\left(k_{p} d\right)$ and a steepness of $A_{L} k_{p}=0.3$ : (a) mean wavenumber $\mathcal{K}(t)$ normalised by the initial value $\mathcal{K}_{0}$; (b) RMS spreading parameter $\varsigma(t)$ normalised by the initial value $\varsigma_{0}$. The various depths are indicated by colour: $k_{p} d=5.592,-; k_{p} d=3.142,-; k_{p} d=2.600,-; k_{p} d=2.000$, $; k_{p} d=1.600, \stackrel{2}{\longrightarrow} k_{p} d=1.363$.

case of $k_{p} d=5.592$ depicted in figure 9 (a) exhibits greater energy transfers along the unidirectional $k_{x}$-axis and the Phillips resonance loop than observed for the $k_{p} d=3.142$ case shown in figure 9(b). Thus, the quasi-degenerate interactions appear to be depth sensitive even in water typically considered to be deep; the observation can be explained by the interplay between the modulation instability and the return current as found by Benjamin (1967) and Whitham (1974) and discussed by Dysthe (1979) and Janssen \& Onorato (2007). The return current develops beneath the wave group with a length scale comparable to the wave group as a whole. Thus, the return current is more sensitive to depth than any individual wave component and, as observed in this study, can be altered by depths greater than $k_{p} d=3.142$. The return current is also capable of 'detuning' the modulational instability. Thus, the sensitivity of the return current to depth appears to influence the quasi-degenerate interactions observed in this study, altering the nonlinear wave-wave interactions of cases $k_{p} d=5.592$ and $k_{p} d=3.142$.

In contrast, the spreading parameter shown in figure $8(\mathrm{~b})$ is less significantly affected by depth. The reduction in directional spreading, observed during focus, achieves a similar minimum value for all the finite-depth cases, although the less deep cases exhibit a delay compared with the deeper cases. The delay is expected because the increase in directional spreading is associated with spectral broadening due to quasi-degenerate interactions. Thus, suppression of the quasi-degenerate interactions by depth delays the onset of non-degenerate interactions. The increase in directional spreading, which dominates the wavenumber spectrum after focus, exhibits only a weak sensitivity to depth with an almost identical spreading parameter at the end of the simulation for finite depths $\left(k_{p} d\right)$ of 5.592, 3.142 and 2.600. The relative insensitivity of the spreading parameter to depth, compared with the mean wavenumber, appears to result from differing depth sensitivity for the quasi-degenerate and non-degenerate interactions discussed in section 3.1. As demonstrated by figure 9 , the quasi-degenerate interactions which transfer energy along the unidirectional $k_{x}$-axis and the Phillips resonance loop exhibit a clear sensitivity to depth and continuously weaken for less deep cases, almost disappearing for the case of $k_{p} d=1.363$. In contrast, the non-degenerate interactions, which develop once the wave spectrum is sufficiently broad-banded and transfer energy at an angle of $\pm 55^{\circ}$ to the spectral peak, are significantly less affected by depth. The oblique energy transfers at 


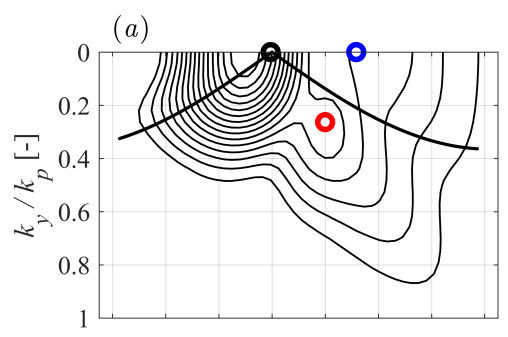

(b)

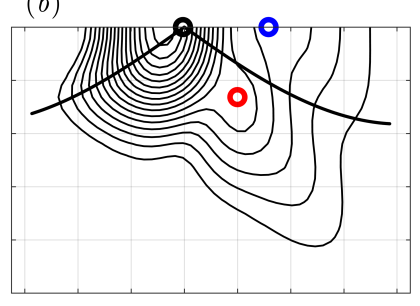

$(e)$ $(c)$

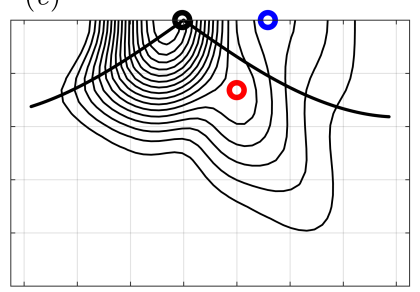

$(f)$

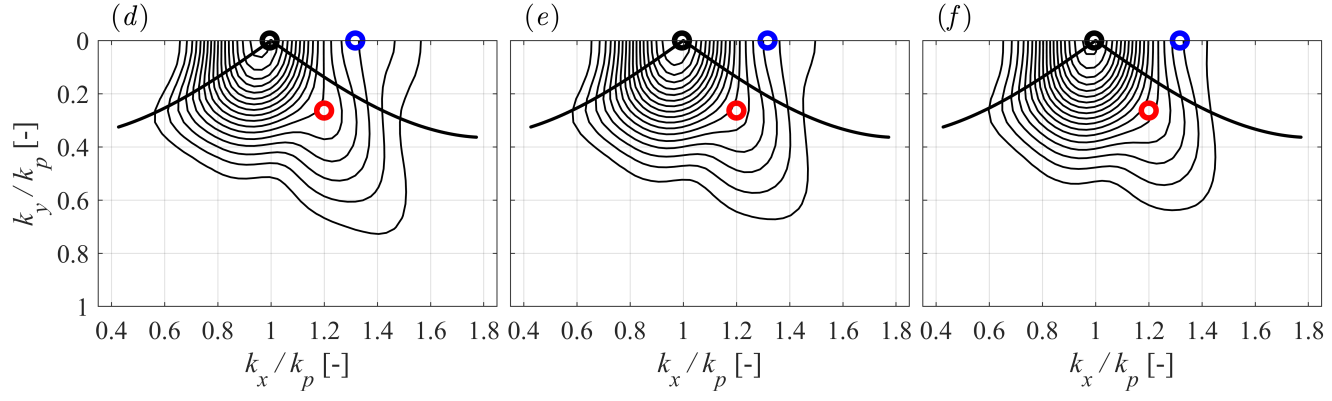

Figure 9. Amplitude spectra of surface elevation at the end of the simulation, $t / T_{0}=15$, corresponding to case GG15 with a steepness of $A_{L} k_{p}=0.3$ and various dimensionless depths: (a) $k_{p} d=5.592 ;(b) k_{p} d=3.142 ;(c) k_{p} d=2.600 ;(d) k_{p} d=2.000 ;(e) k_{p} d=1.600$; (f) $k_{p} d=1.363$. Contour levels $(-)$ are evenly distributed between $0.01 \mathrm{~m}$ and 0.105 $\mathrm{m}$ in intervals of $0.005 \mathrm{~m}$. The thick black line ( $)$ represents segments of the Phillips 'figure-of-eight' resonance loop and the open circles specify particular wavenumber components: $\mathcal{Q}(\mathbf{O}) k_{x} / k_{p}=0.995, k_{y} / k_{p}=0.000 ; \mathcal{R}(\mathbf{O}) k_{x} / k_{p}=1.200, k_{y} / k_{p}=0.263 ; \mathcal{S}(\mathbf{O}) k_{x} / k_{p}=1.317$, $k_{y} / k_{p}=0.000$.

$\pm 55^{\circ}$ are suppressed by depth but continue to dominate the spectral evolution even for $k_{p} d=1.363$. Consequently, the mean wavenumber is sensitive to energy transfers to higher wavenumbers by the quasi-degenerate interactions and, thus, exhibits depth sensitivity. The spreading parameter is principally governed by the oblique energy transfers of the non-degenerate interactions and, thus, exhibits less sensitivity to depth.

We have also analysed the change in wave action density for components $\mathcal{R}$ and $\mathcal{S}$ with the results shown in figure 10. Normalisation is performed with the initial wave action density of the spectral peak $\left(\mathcal{Q}_{0}\right)$. The wave action density of component $\mathcal{S}$, shown in figure 10(a), depicts a qualitatively similar trend for all the depth cases but demonstrates suppressed energy transfers at reduced depths. In contrast, component $\mathcal{R}$ depicts a similar quantitative trend in wave action density for all depths and even exhibits faster growth for finite depth cases $k_{p} d=2.000$ and $k_{p} d=1.600$ than the deep water cases. Benney \& Roskes (1969) and McLean (1982a) showed that the peak instability of a regular wave train becomes oblique, rather than unidirectional, at intermediate depths. Thus, the faster growth rate for component $\mathcal{R}$ observed for cases $k_{p} d=2.000$ and $k_{p} d=1.600$ may be a result of the quasi-degenerate interactions biasing towards oblique rather than unidirectional components. Ultimately, depth suppresses all forms of spectral evolution in the intermediate range of depths $\left(5.592>k_{p} d>1.363\right)$ considered by this study, consistent with the finite-depth random sea simulations of Toffoli et al. (2009). However, unidirectional energy transfers appear to be particularly suppressed relative to the oblique energy transfers. Thus, the increase in mean wavenumber is suppressed, which suggests less severe wave kinematics. However, the reduction in directional spreading, observed during focus, appears to occur for all the finite-depth cases suggesting that the 


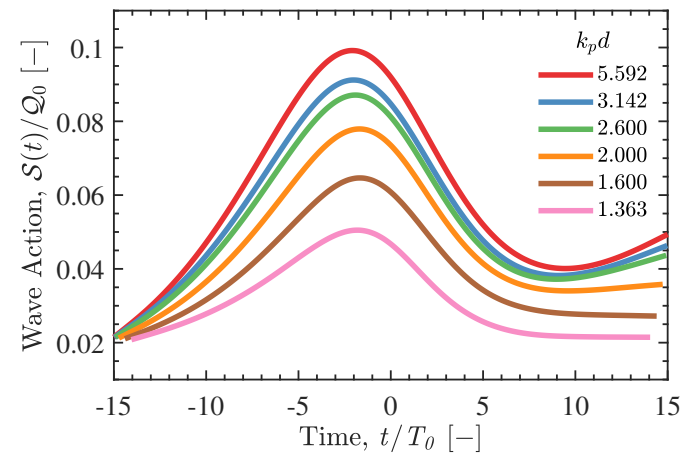

(a)

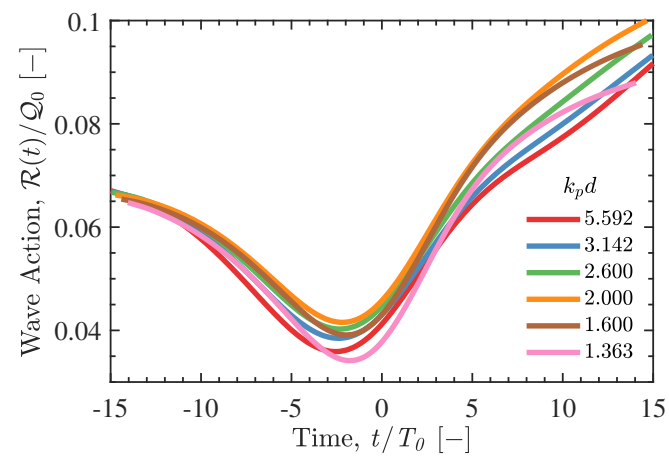

(b)

Figure 10. Change in wave action density corresponding to case GG15 with a steepness of $A_{L} k_{p}=0.3$ and various dimensionless depths: $k_{p} d=5.592, \quad ; k_{p} d=3.142$, ; $k_{p} d=2.600,-; k_{p} d=2.000,-; k_{p} d=1.600,-; k_{p} d=1.363,=$. The analysis has been performed for specific wavenumber components: (a) component $\mathcal{S}\left(k_{x} / k_{p}=1.317\right.$, $\left.k_{y} / k_{p}=0.000\right) ;(\mathrm{b})$ component $\mathcal{R}\left(k_{x} / k_{p}=1.200, k_{y} / k_{p}=0.263\right)$; both components are normalised by the initial wave action density of the spectral peak $\left(\mathcal{Q}_{0}\right)$, and the location of the components in the wavenumber spectrum is shown in figure 9 .

'wall of water' effect may also be observed in waters of intermediate depth, consistent with the experiments of Latheef et al. (2017). The interplay between the return current and modulation instability also results in depth sensitivity within the range of depths commonly considered to be 'deep', suggesting that the length scale of the return current and wave group are of greater significance than the length scale of individual wave components when categorising water as 'deep'.

\subsubsection{Directional spreading}

We consider the effects of directional spreading for wave groups with a linear steepness $\left(A_{L} k_{p}\right)$ of 0.24 based on a Gaussian wavenumber magnitude spectrum with a Gaussian spreading function and a finite depth $\left(k_{p} d\right)$ of 3.142. Our simulations include initial spreading parameters $\left(\varsigma_{0}\right)$ in the range of $5^{\circ}$ to $25^{\circ}$ in intervals of $5^{\circ}$. Focused wave events with high values of directional spreading exhibit a reduced focal time, compared with less-spread or unidirectional events, due to linear dispersion. Figure 11(a) shows the steepness of the wave envelope for the various cases of directional spreading. As can be seen, the less spread cases, e.g. $\varsigma_{0}=10^{\circ}$, begin with a higher steepness and steepen gradually until focus. In contrast, the cases with more directional spreading, e.g. $\varsigma_{0}=25^{\circ}$, begin with a lower steepness and steepen rapidly before focus. Directional spreading, thus, reduces the focal time of the wave event, limiting the total energy transfer in an integral sense. Figure 11(a) also demonstrates the influence of nonlinear interactions on the life span of the focused wave events. Under linear evolution, each case should focus and defocus symmetrically, see figure 2. However, cases $\varsigma_{0}=5^{\circ}$ and $\varsigma_{0}=10^{\circ}$ exhibit deviations between focusing and defocusing due to nonlinear interactions, extending the life span of the focused events.

The spectral evolution is also demonstrated by figure 11(b), which shows the zeroth moment of the amplitude spectrum $\left(\mathcal{M}_{0}\right)$, normalised by the linear amplitude at focus $\left(A_{L}\right)$, for all the spreading cases. Under linear evolution, $\mathcal{M}_{0}=A_{L}$ throughout the simulation. Under nonlinear evolution, an increase in $\mathcal{M}_{0}$ indicates a transition from a narrow-banded to a more broad-banded spectrum. The zeroth moment increases drastically for the $\varsigma_{0}=5^{\circ}$ case due to rapid broadening of the spectrum. Consequently, 


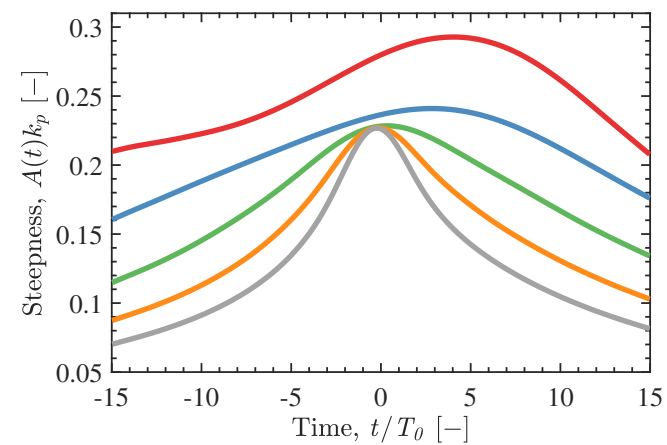

(a)

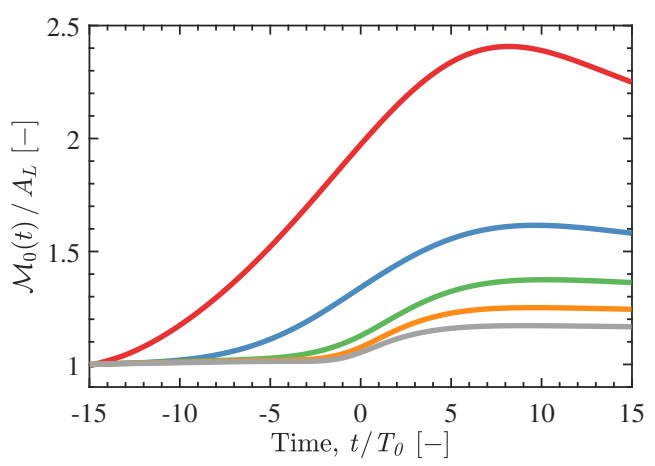

(b)

FIGURE 11. Effect of directional spreading for wave groups with a steepness of $A_{L} k_{p}=0.24$, finite depth of $k_{p} d=3.142$ and initial spreading parameters of $5^{\circ}(-), 10^{\circ}(-), 15^{\circ}(-)$, $20^{\circ}(-)$ and $25^{\circ}(-)$ : (a) Steepness of the wave envelope over time - the amplitude $A(t)$ is based on the maximum elevation of the wave envelope at time $t ;(b)$ The zeroth moment of the wavenumber-amplitude spectrum $\left(\mathcal{M}_{0}\right)$ normalised by the linear focus amplitude $\left(A_{L}\right)$. All cases are based on a Gaussian wavenumber spectrum with a Gaussian spreading function.

the focused wave event is significantly steeper than the linear steepness, and the lifespan of the event is extended. In contrast, the $\varsigma_{0}=25^{\circ}$ case depicts a nearly constant zeroth moment until shortly before focus, indicating less spectral broadening during focus, which results in a steepness and focused lifespan which resembles the linear event.

The associated change in mean wavenumber $\mathcal{K}(t)$ is shown in figure $12(\mathrm{a})$ for the various cases of directional spreading, normalised by the initial value $\mathcal{K}_{0}$. The increase in mean wavenumber is also delayed for cases with more directional spreading due to the comparatively late focusing of the event, but the qualitative trend is consistent. An increase in directional spreading reduces energy transfers and limits the growth in mean wavenumber. However, the change in spreading parameter shown in figure 12(b) depicts a qualitatively different trend for the different cases of directional spreading. Case $\varsigma_{0}=5^{\circ}$ features an increase in directional spreading during focus and ultimately results in a focused event which is more spread than the initial condition - the time of nonlinear focus is indicated with a black hexagram. Case $\varsigma_{0}=10^{\circ}$ features a reduction in directional spreading for a few wave periods after the start of the simulation but thereafter an increase in directional spreading follows and the focused wave event is also more spread than the initial conditions. Conversely, cases $\varsigma_{0}=15^{\circ}, 20^{\circ}$ and $25^{\circ}$ feature a reduction in directional spreading before focus and the focused wave event is ultimately less spread than the initial conditions.

These results suggest that a steep wave event in a sea with low directional spreading, i.e., $\varsigma_{0} \leqslant 10^{\circ}$, may tend to be more spread than the background sea state, while seas with more directional spreading, i.e., $\varsigma_{0} \geqslant 15^{\circ}$, may produce steep wave events which are less directionally spread than the background sea state. Note, the cases with more directional spreading, $\varsigma_{0} \geqslant 15^{\circ}$, exhibit a reduction in spreading before focus, but the trend is largely reversed after focus so the final value of the spreading parameter nearly matches the initial value, as demonstrated by case $\varsigma_{0}=25^{\circ}$. However, the cases with low spreading $\varsigma_{0} \leqslant 10^{\circ}$ do not exhibit such a reversal - the final spreading parameter is significantly larger than the initial spreading parameter. 


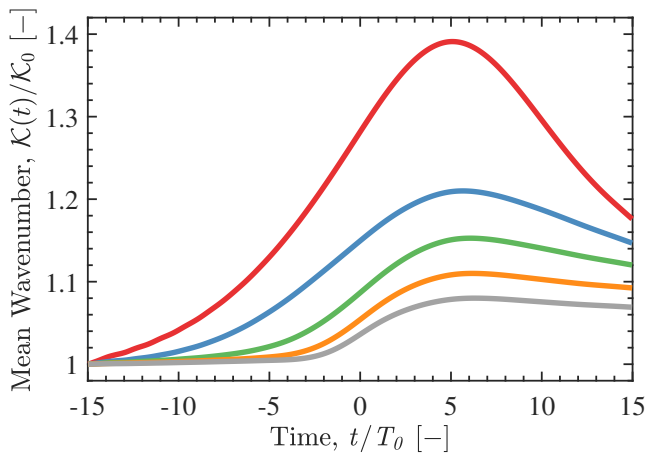

(a)

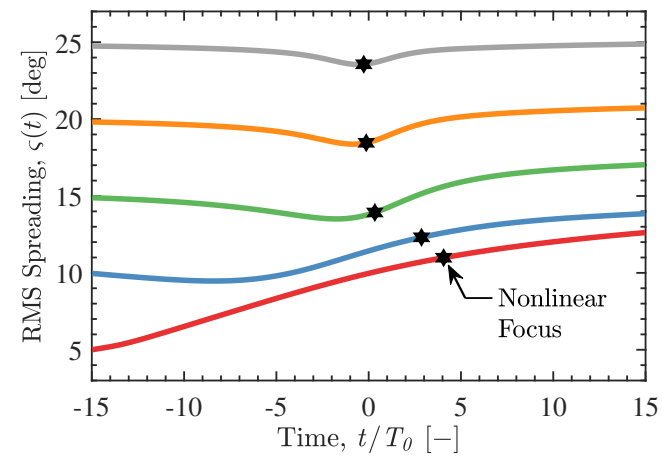

(b)

FiguRE 12. Spectral evolution over time for wave groups with a steepness of $A_{L} k_{p}=0.24$, dimensionless depth of $k_{p} d=3.142$ and and initial spreading parameters of $5^{\circ}(=), 10^{\circ}$ $(-), 15^{\circ}(-), 20^{\circ}(-)$ and $25^{\circ}(-)$. The spectral parameters include: (a) mean wavenumber $\mathcal{K}(t)$ normalised by the initial value $\mathcal{K}_{0}$; (b) RMS spreading parameter $\varsigma(t)$. The time of nonlinear focus is indicated with a black hexagram. All cases are based on a Gaussian wavenumber spectrum with a Gaussian spreading function.

\subsection{Effect of the high-wavenumber tail}

The peak of the JONSWAP spectrum contains most of the total energy. Thus, Gaussian wavenumber spectra are frequently used in studies of wave-wave interactions with the high-wavenumber components of the tail neglected from the analysis (see, e.g., Mori et al. (2011)). Certain physical processes have been found to suppress high-wavenumber components, which comprise the tail of the spectrum, more severely than low-wavenumber components. Such mechanisms can suppress the development of the high-wavenumber tail. Wave blocking due to currents (see, e.g., Ma et al. (2010)) can suppress the tail of the wave spectrum at a particular location, if low-wavenumber components continue to propagate while high-wavenumber components are blocked from propagating further, as discussed by Chawla \& Kirby (2002). Rapizo et al. (2016) found that the development of the spectral tail is disturbed for waves propagating on a coflowing current, due to detuning of the quartet resonance interactions. Similarly, Waseda et al. (2015) found that the spectral tail can be suppressed by detuning due to currents. Ice sheets also tend to suppress high-wavenumber components, attenuating the amplitude of highwavenumber components more severely than low-wavenumber components (see Meylan et al. (2018) and Toffoli et al. (2015)), possibly suppressing the tail of the spectrum. Thus, a comparison between Gaussian and JONSWAP spectra serves two purposes: (1) to indicate the appropriacy of a Gaussian spectrum as a model for the peak of a JONSWAP spectrum in typical sea states and; (2) to contrast the spectral evolution of a steep wave event in seas with and without a fully-developed spectral tail.

We combine a JONSWAP wavenumber spectrum $(\gamma=3.3)$ with a frequencyindependent spreading function, a Gaussian with an initial spreading parameter of $\varsigma_{0}=15^{\circ}$, and denote this case as JG15. Similarly, we combine a JONSWAP spectrum $(\gamma=3.3)$ with the frequency-dependent spreading function of Ewans (1998) and denote this case as JE. The combination of a JONSWAP spectrum with an Ewans (1998) spreading function realistically captures the spectral shape of fetch-limited seas. We compare against two cases based on a Gaussian wavenumber spectrum: one with an initial spreading parameter of $\varsigma_{0}=15^{\circ}$, denoted as GG15, and another with an initial spreading parameter of $\varsigma_{0}=20^{\circ}$, denoted as GG20. A summary of the case acronyms 


\begin{tabular}{l|c|c|c} 
Case & Wavenumber & Spreading & Spreading \\
& $S(k)$ & Function, & Parameter, \\
GG5 & Gaussian & Gaussian & $\varsigma_{0}$ \\
GG10 & Gaussian & Gaussian & $10^{\circ}$ \\
GG15 & Gaussian & Gaussian & $15^{\circ}$ \\
GG20 & Gaussian & Gaussian & $20^{\circ}$ \\
GG25 & Gaussian & Gaussian & $25^{\circ}$ \\
JG15 & JONSWAP & Gaussian & $15^{\circ}$ \\
JE & JONSWAP & Ewans (1998) & -
\end{tabular}

TABLE 4. Summary of acronyms for simulation cases.

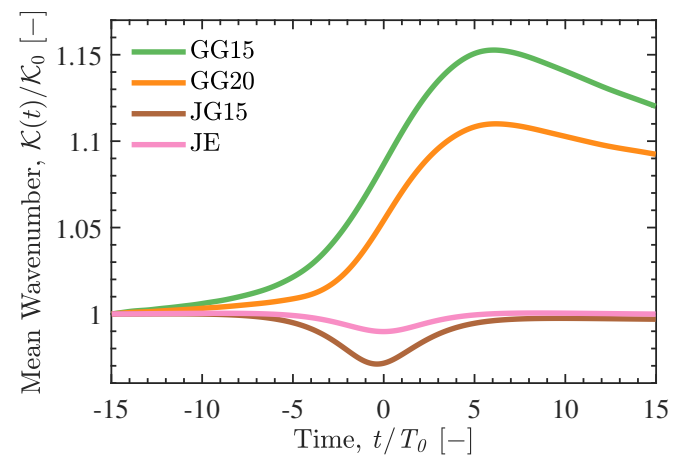

(a)

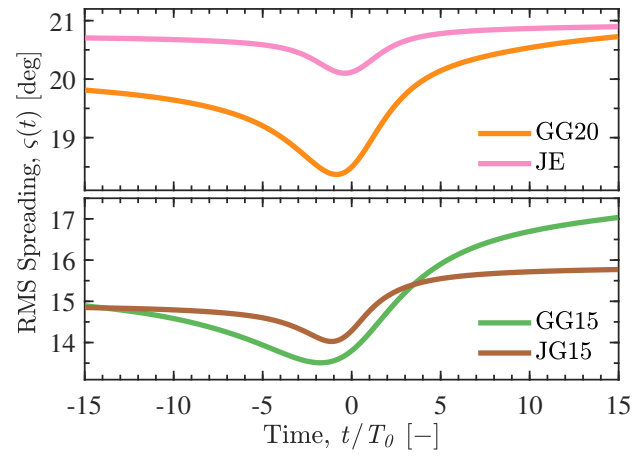

(b)

Figure 13. The effect of the high-wavenumber tail on the spectral evolution of wave groups with a steepness of $A_{L} k_{p}=0.24$, dimensionless depth of $k_{p} d=3.142$ and different spectral shapes: GG15, - ; GG20, - JG15, - JE, - . The spectral parameters include: (a) mean wavenumber $\mathcal{K}(t)$ normalised by the initial value $\mathcal{K}_{0}$; (b) RMS spreading parameter $\varsigma(t)$ in degrees.

is listed in table 4 . The results are shown in figure 13 for both the mean wavenumber $\mathcal{K}(t)$ and spreading parameter $\varsigma_{0}(t)$. The mean wavenumber is normalised by the initial value for each case $\mathcal{K}_{0}$. Figure $13(\mathrm{a})$ indicates that the simulations performed with a Gaussian wavenumber spectrum produces an increase in mean wavenumber. However, the simulations performed with a JONSWAP spectrum, cases JG15 and JE, exhibit a qualitatively different trend; the mean wavenumber remains approximately constant during the early stages of focusing and reduces during the focus event. Thus, the increase in mean wavenumber observed for the Gaussian cases, GG15 and GG20, appears to be associated with redevelopment of the truncated high-wavenumber tail, and the JONSWAP cases do not show the same trend. The trend for the spreading parameter is however qualitatively similar between the Gaussian and JONSWAP cases, as shown in figure 13(b). All cases feature a reduction in the spreading parameter during focus, indicating focused wave events which are less directionally spread than the background sea state, i.e. the 'wall-of-water' effect.

Thus, a Gaussian wavenumber spectrum exhibits qualitatively similar trends to a JONSWAP spectrum in terms of the spreading parameter, but redevelopment of the 


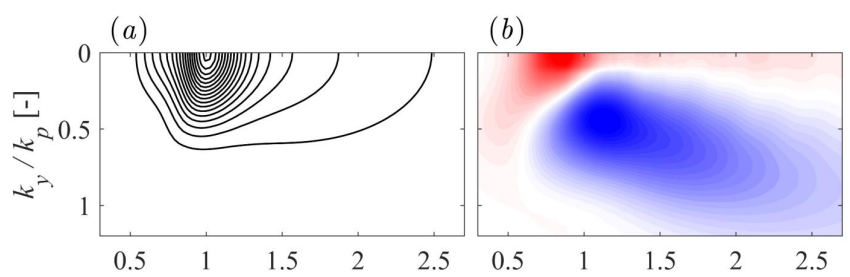

(c) $\times 10^{-3}$
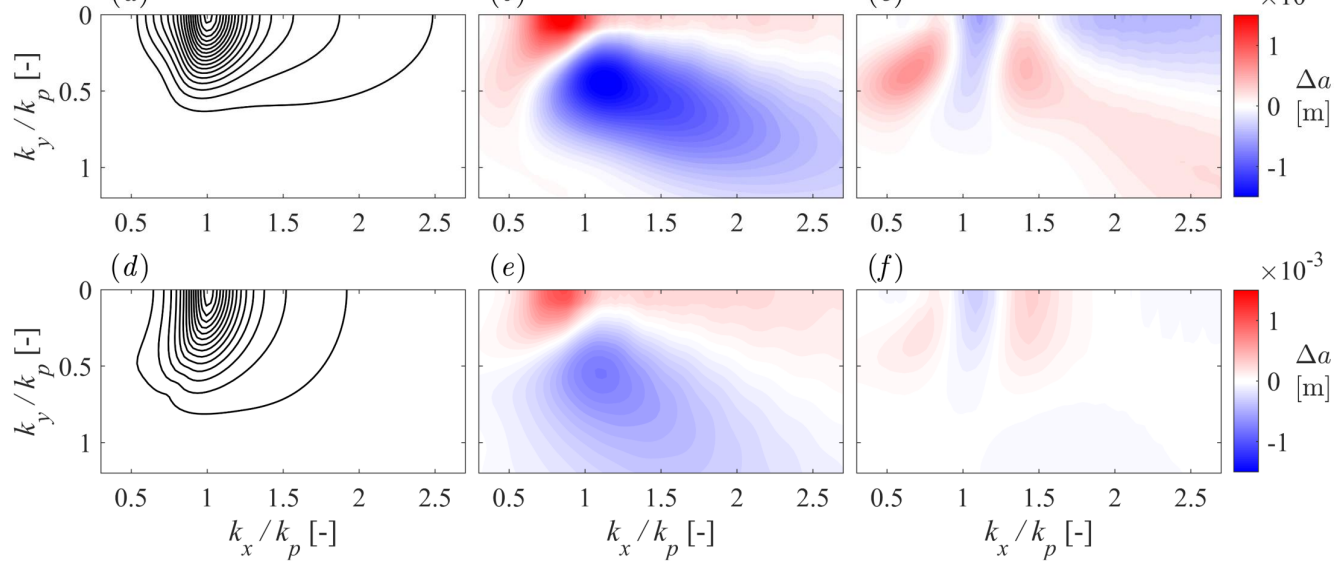

(e)

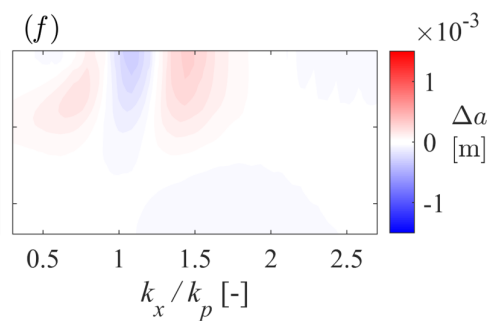

FiguRE 14. Amplitude spectra of surface elevation for JONSWAP cases: JG15 in $(a),(b),(c)$; and JE in $(d),(e),(f)$. Initial conditions $\left(t / T_{0}=-15\right)$ are shown in $(a)$ and $(d)$ with contour levels $(-)$ evenly distributed between $0.002 \mathrm{~m}$ and $0.034 \mathrm{~m}$ in intervals of $0.002 \mathrm{~m}$. The difference in the amplitude spectrum at time $t / T_{0}=0$, relative to the initial condition, is shown in $(b)$ and (e) using a colour scale with levels shown in the figure. Similarly, the difference in the amplitude spectrum at the end of the simulation $\left(t / T_{0}=15\right)$, relative to the initial condition, is shown in $(c)$ and $(f)$ using the same colour scale.

spectral tail leads to an increase in mean wavenumber, which is not observed for the JONSWAP spectrum. Furthermore, the changes in mean wavenumber and spreading parameter for the JONSWAP cases appear to be largely temporary since the reduction in both parameters reverses after focus. In contrast, the changes in mean wavenumber and spreading parameter for the Gaussian cases appear to include more permanent changes since the trends do not entirely reverse after focus. Thus, the JONSWAP spectra exhibit a form of spectral equilibrium which inhibits changes to the spectrum. The results of the previous sections based on Gaussian wavenumber spectra may, thus, be most applicable to sea states without a fully developed spectral tail. Energy transfer to higher wavenumbers, in particular, is reduced for steep wave events based on a fully-developed JONSWAP spectrum. We conclude that the high-wavenumber tail of the spectrum influences the spectral evolution of a steep wave event. Thus, studies based solely on Gaussian spectra, e.g. Adcock et al. (2015), should be treated with caution.

Amplitude spectra of surface elevation, shown in figure 14, depict the spectral evolution of the JONSWAP cases (JG15 and JE). The top row of panels, figure $14(a, b, c)$, correspond to case JG15. The bottom row of panels, figure $14(d, e, f)$, correspond to case JE. The initial conditions $\left(t / T_{0}=-15\right)$ are shown in figure 14 $(a)$ and figure $14(d)$. The initial conditions indicate that the Ewans (1998) spreading function of case JE features higher levels of spreading in the tail than the Gaussian spreading function of case JG15. Figure 13(b) confirms that the spreading parameter is consistently lower for case JG15 than case JE, due to lower levels of spreading in the tail for case JG15. The spectral evolution of both JONSWAP cases (JG15 and JE) is less noticeable than the GG cases. Thus, we show the difference in the amplitude spectrum relative to the initial condition: around the time of focus $\left(t / T_{0}=0\right)$ in figure $14(b, e)$; and at the end of the simulation $\left(t / T_{0}=15\right)$ in figure $14(c, f)$. We use a colour scale to indicate the growth (in red) and decline (in blue) of component amplitudes relative to the initial condition. As shown in figure 13, the spectral changes are most apparent around the time of focus $\left(t / T_{0}=0\right)$, figure $14(b, e)$, but the changes appear to be mostly temporary and reverse after focus, leaving few permanent 
changes at the end of the simulation $\left(t / T_{0}=15\right)$, figure $14(c, f)$. Case JG15 does exhibit some permanent changes to the spectrum - the directional spreading of the tail increases as energy is transferred to oblique high-wavenumber components, consistent with the net increase in the spreading parameter shown in figure 13(b). In contrast, case JE exhibits fewer permanent changes, consistent with the results of figure 13. Thus, high levels of directional spreading in the tail, e.g. Ewans (1998), may also be a feature of spectral equilibrium. Case JG15 also exhibits more substantial spectral changes around the time of focus $\left(t / T_{0}=0\right)$, figure $14(b, e)$. Contraction of the spectrum in the $k_{y}$-direction (the 'wall-of-water' effect) and a downshift in the spectral peak occurs for both case JG15 and case JE. However, both effects are more substantial for case JG15, providing further evidence that case JE may be a better representation of spectral equilibrium than case JG15 and directional spreading in the tail can influence spectral evolution.

\subsection{Downshift of the spectral peak}

We observe a downshift in wavenumber for the spectral peak during our simulations, see figure 3 as an example. First reported by Lake et al. (1977), the phenomenon is commonly termed a frequency/wavenumber downshift and has been observed by numerous studies (see Ma et al. (2010), Kharif et al. (2008a), Segur et al. (2005), Dysthe et al. (2003), Melville (1982), Su et al. (1982) and others). Downshifting has been related to nonlinear wave-wave interactions (e.g., Trulsen \& Dysthe (1997)), breaking (e.g., Trulsen \& Dysthe (1990), Tulin \& Waseda (1999)) and wind (e.g., Hara \& Mei (1991)). The downshift we observe in our potential flow simulations is exclusively due to nonlinear wave-wave interactions. We base our analysis on component $\mathcal{Q}\left(k_{x} / k_{p}=1.000, k_{y} / k_{p}=0.000\right)$, which coincides initially with the peak of the wavenumber spectrum. The rate of change in wave action density for $\mathcal{Q}$ is indicative of the rate of downshift for the spectral peak, and we consider the influences of directional spreading and the high-wavenumber tail on the downshift, with the results shown in figure 15. We consider Gaussian wavenumber spectra with a Gaussian spreading function and initial spreading parameters $\left(\varsigma_{0}\right)$ of $5^{\circ}, 10^{\circ}, 15^{\circ}$, $20^{\circ}, 25^{\circ}$; the cases are denoted as GG5, GG10, GG15, GG20 and GG25, respectively. For comparison, we consider a JONSWAP spectrum $(\gamma=3.3)$ with frequency-independent spreading, based on a Gaussian spreading function with $\varsigma_{0}=15^{\circ}$, and denote this case as JG15. We also consider a JONSWAP spectrum with frequency-dependent spreading, based on the Ewans (1998) spreading function, and denote this case as JE. A linear steepness at focus $\left(A_{L} k_{p}\right)$ of 0.24 has been selected for all cases together with a depth $\left(k_{p} d\right)$ of 3.142 .

The growth rate for component $\mathcal{Q}$ is shown in figure 15 for the various cases together with the dynamic timescale. For all cases except GG5, the wave action of component $\mathcal{Q}$ initially increases before the downshift of the peak begins (see figure 4 (a) as an example). The growth rate is shown in absolute terms. Thus, a discontinuity is shown in the logarithmic plots, at the time of maximum wave action density, when the growth rate momentarily reaches zero before a change in sign occurs. The time of nonlinear focus for each case is indicated with a black hexagram in figure 15, and the time interval for which the steepness of the wave envelope $A(t) k_{p}$ exceeds 0.18 is indicated by a magenta line segment. The growth rate for case GG5 is augmented because the steepness of the event exceeds the linear steepness, used to calculate the dynamic growth rate, as evidenced by figure 11(a). The other GG cases achieve a steepness at focus which is comparable to the linear steepness, and the post-focus growth rate for component $\mathcal{Q}$ can be seen in figure 15 to be consistent with the dynamic timescale. However, an increase in directional spreading for the GG cases does reduce the rate of downshift for the spectral peak and 

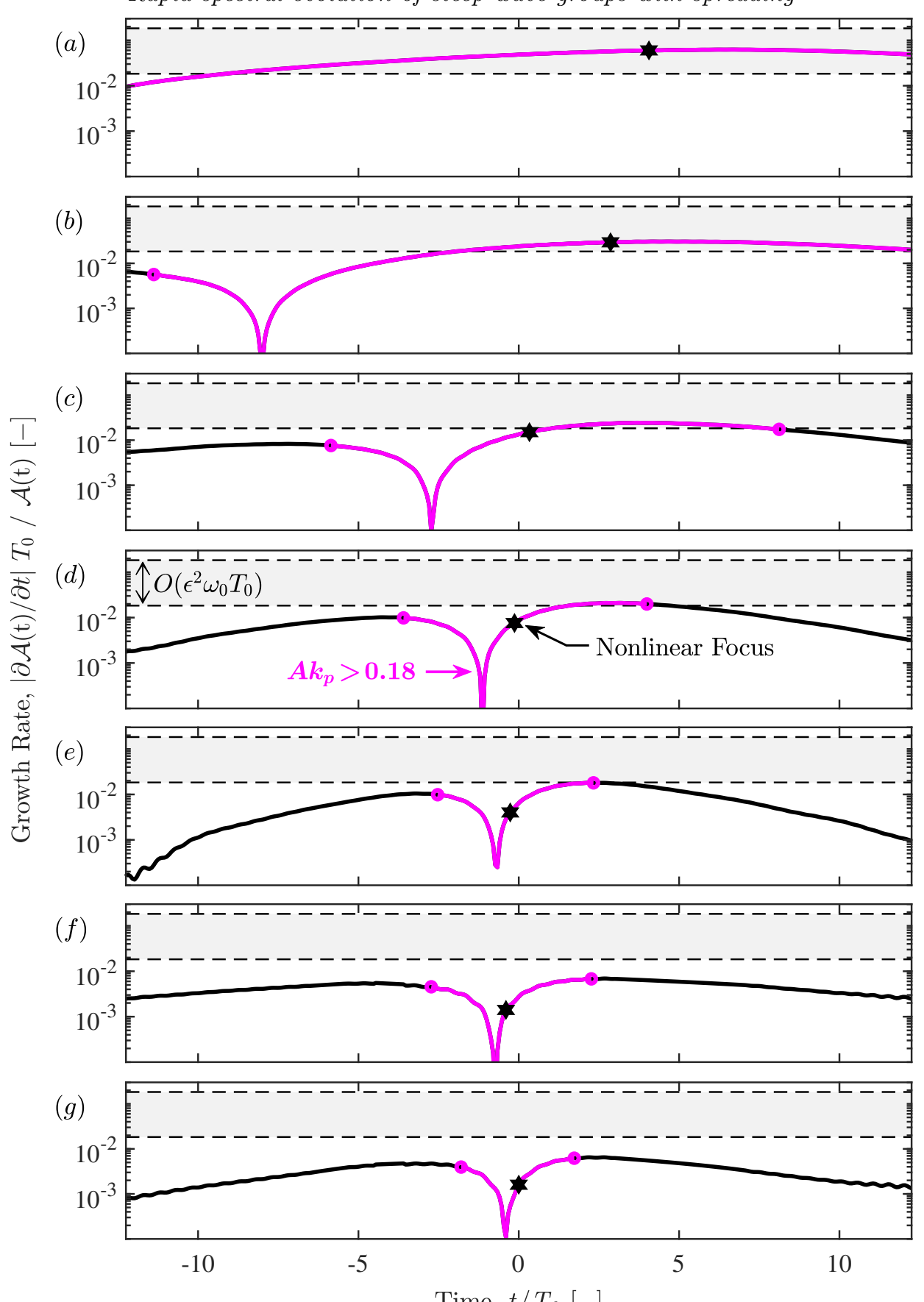

Time, $t / T_{0}[-]$

Figure 15. Growth rate of wave action density $\mathcal{A}$ for the spectral component $\mathcal{Q}$, which initially coincides with the peak of the amplitude spectrum: (a) GG5; (b) GG10; (c) GG15; (d) GG20; (e) GG25; $(f) \mathrm{JG} 15 ;(g) \mathrm{JE}$. The dynamic growth rate $O\left(\epsilon^{2} \omega_{0} T_{0}\right)$ is indicated by bounded $(---)$ grey bands. The magenta line segment $(-)$ indicates the time interval during which $A(t) k_{p}$, as shown in figure 11 , exceeds 0.18 . The value of $\epsilon$ equates to $A_{L} k_{p} / \pi$ in the calculation of the growth rates. The time of nonlinear focus is indicated with a hexagram. 


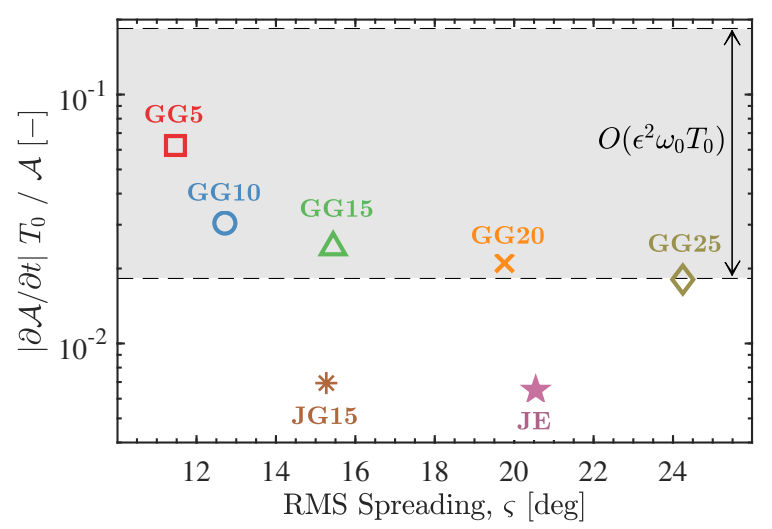

FIGURE 16. The stabilising effect of directional spreading and the high-wavenumber tail: the vertical axis indicates the maximum growth rate calculated at any point in the simulation; the horizontal axis indicates the RMS spreading parameter of the spectrum at the corresponding time. The growth rate calculation is based on component $\mathcal{Q}\left(k_{x} / k_{p}=0.995, k_{y} / k_{p}=0.000\right)$, which initially coincides with the peak of the wavenumber spectrum. The various cases, all with a steepness of $A_{L} k_{p}=0.24$ and a depth of $k_{p} d=3.142$, are labelled within the figure. The dynamic growth rate $O\left(\epsilon^{2} \omega_{0} T_{0}\right)$ is indicated by bounded $(---)$ grey bands. The value of $\epsilon$ equates to $A_{L} k_{p} / \pi$ in the growth rate calculations.

the inclusion of the high-wavenumber tail of the spectrum, featured in the JG15 and JE cases, leads to a further decrease.

The effect of the high-wavenumber tail on the downshift is summarised in figure 16, which shows the maximum growth rate, at any time in the simulation, on the vertical axis. The horizontal axis shows the corresponding spreading parameter $(\varsigma(t))$, at the time that the maximum growth rate occurs. Cases GG15 and JG15 exemplify the role of the high-wavenumber tail in reducing the downshift phenomenon; the two cases exhibit a similar spreading parameter but the maximum growth rate for case GG15 is a factor of three faster than case JG15. The slower growth rate for case JG15 is particularly noteworthy because the JG15 focused wave event is arguably steeper than the GG15 event. The linear steepness is calculated from $A_{L} k_{p}$, which incorporates the spectral peak, at the start of the simulation, as the characteristic wavenumber. A Gaussian wavenumber spectra is symmetric about the spectral peak which supports the use of $k_{p}$ as the characteristic wavenumber, particularly if the spectrum is narrow-banded. However, a JONSWAP spectrum features a high-wavenumber tail which arguably should result in a characteristic wavenumber above $k_{p}$, suggesting cases JG15 and JE are steeper than indicated by $A_{L} k_{p}$. However, the maximum growth rate for case GG15 is significantly higher than the maximum growth rate for cases JG15 and JE. Inclusion of the highwavenumber tail, thus, appears to reduce the downshift of the spectral peak.

In summary, all focused wave events in this study exhibit a downshift of the spectral peak. However, spreading and the inclusion of the high-wavenumber tail reduce the downshift, and nonlinear evolution of the wavenumber spectrum in general. The mechanism is not limited to a reduced focal time caused by linear dispersion. Rather, the rate of the downshift, even at the steepest stages of focusing, is also reduced by spreading and the high-wavenumber tail. Thus, directional spreading and the spectral tail appear to be integral features of a spectral equilibrium, which limits directional energy transfers during a steep wave event. 


\section{Conclusion}

We have studied steep focusing wave groups formed by dispersive focusing in deep and intermediate waters. For narrow-banded Gaussian wavenumber spectra with a Gaussian spreading function, quasi-degenerate interactions result in directional energy transfers: (1) Along the $k_{x}$-axis, consistent with the unidirectional instabilities observed by Longuet-Higgins (1978) and McLean (1982b) for a regular wave; (2) At angles of $\pm 35^{\circ}$ to the spectral peak with a bias towards high-wavenumber components, consistent with the $\arctan (1 / \sqrt{2})$ resonance angle identified by Longuet-Higgins (1976) and the Phillips 'figure-of-eight' resonance loop.

Spectral broadening due to the quasi-degenerate interactions eventually facilitates nondegenerate interactions, which dominate the spectral evolution of the wave group after focus. The non-degenerate interactions manifest as high-wavenumber sidelobes at angles of $\pm 55^{\circ}$ to the spectral peak. We also observe contraction of the wavenumber spectrum in the $k_{y}$-direction during focusing and a downshift in wavenumber for the spectral peak, consistent with previous studies (see, e.g., Trulsen \& Dysthe (1997)). All the spectral changes we observe can be attributed to quartet interactions. Relative to linear theory, the combination of quasi-degenerate and non-degenerate interactions results in nonlinear wave events with augmented kinematics and an extended lifespan.

Finite depth weakens all forms of spectral evolution. However, the quasi-degenerate interactions exhibit a greater sensitivity to depth, indicating suppression of the modulation instability by the return current as discussed by Dysthe (1979) and Janssen \& Onorato (2007). We also observe sensitivity to depth for $k_{p} d$ values commonly considered to be deep, $3.142<k_{p} d<5.592$. The non-degenerate interactions appear to be significantly less suppressed by depth with persistent evidence of a $\pm 55^{\circ}$ spectral sidelobe at a depth of $k_{p} d=1.363$. Although the quasi-degenerate interactions are significantly suppressed by depth, the interactions do not entirely disappear for $k_{p} d=1.363$ and show signs of biasing towards oblique rather than unidirectional wave components at intermediate depths, consistent with McLean (1982a). The contraction of the wavenumber spectrum in the $k_{y}$-direction has also proved to be resilient to depth, suggesting that lateral expansion of the wave group and the 'wall-of-water' effect may persist at intermediate depths.

We contrast our analyses of Gaussian cases with simulations based on a JONSWAP spectrum, combined with frequency-independent and frequency-dependent spreading, and find that the high-wavenumber tail of the spectrum reduces the nonlinear features observed for the Gaussian cases. In particular, the energy transfers to higher wavenumbers is reduced for the JONSWAP cases, suggesting that the Gaussian simulations are influenced by the absence of a spectral tail and the nonlinear interactions bias towards redevelopment of the tail. However, the trends in the spreading parameter show qualitative agreement between the Gaussian and JONSWAP cases. We conclude that focused wave events in sea states without a fully developed spectral tail are more likely to exhibit rapid nonlinear evolution. Physical mechanisms which can suppress the highwavenumber components in the spectral tail, such as dissipation by ice sheets (see Meylan et al. (2018)) and wave blocking by currents (see Chawla \& Kirby (2002)), could thus influence the characteristics of focused wave events in the immediate vicinity. We also consider the downshift of the spectral peak and find that the downshift, and the spectral evolution in general, is reduced by directional spreading and the presence of the highwavenumber tail. Reduced spectral evolution is not only due to the reduced focal time of the more broad-banded events. Rather, the rates of energy transfer also appear to be reduced even at the steepest stages of wave group focusing. Thus, directional spreading 
and the high wavenumber tail appear to be integral features of a spectral equilibrium which reduces the rapid directional energy transfers of a steep wave group.

\section{Acknowledgement}

The authors gratefully acknowledge funding from the DeRisk project of Innovation Fund Denmark (grant number 4106-00038B). TSvdB acknowledges a Royal Academy of Engineering Research Fellowship. DB has been supported by a studentship from the Engineering and Physical Sciences Research Council (EPSRC) of the UK Government and would like to specifically thank Peter S. Tromans of Ocean Wave Engineering Ltd. as well as Alexander V. Babanin and Qingxiang Liu of the University of Melbourne for useful discussions about this work.

\section{Declaration of interests}

The authors report no conflict of interest.

\section{REFERENCES}

Adcock, T. A. A. \& TAYlor, P. H. 2014 The physics of anomalous ('rogue') ocean waves. Rep. Prog. Phys. 77, 105901.

Adcock, T. A. A. \& TAYlOR, P. H. 2016 Fast and local non-linear evolution of steep wavegroups on deep water: A comparison of approximate models to fully-nonlinear simulations. Phys. Fluids 28, 016601.

Adcock, T. A. A., Taylor, P. H. \& Draper, S. 2015 Nonlinear dynamics of wave-groups in random seas: unexpected walls of water in the open ocean. Proc. R. Soc. A 471, 20150660.

Alber, I. E. 1978 The effects of randomness on the stability of two-dimensional surface wavetrains. Proc. R. Soc. A 363, 525-546.

Alberello, A., Chabchoub, A., Monty, J. P., Nelli, F., Lee, J. H., Elsnab, J. \& TOFFoli, A. 2018 An experimental comparison of velocities underneath focussed breaking waves. Ocean Eng. 155, 201-210.

Annenkov, S. Y. \& ShriRA, V. I. 2006 Role of non-resonant interactions in the evolution of nonlinear random water wave fields. J. Fluid Mech. 561, 181-207.

Annenkov, S. Y. \& SHRIRA, V. I. 2018 Spectral evolution of weakly nonlinear random waves: kinetic description versus direct numerical simulations. J. Fluid Mech. 844, 766-795.

BAnner, M. L. \& Young, I. R. 1994 Model spectral dissipation in the evolution of wind waves. Part I: Assessment of existing model performance. J. Phys. Oceanogr. 24 (7), 1550-1571.

Barratt, D., Bingham, H. B. \& Adcock, T. A. A. 2020 Nonlinear evolution of a steep, focusing wave group in deep water simulated with OceanWave3D. J. Offshore Mech. Arct. Eng. 142, 021201.

Barthelemy, X., Banner, M. L., Peirson, W. L., Fedele, F., Allis, M. \& Dias, F. 2018 On a unified breaking onset threshold for gravity waves in deep and intermediate depth water. J. Fluid Mech. 841, 463-488.

Benjamin, T. B. 1967 Instability of periodic wavetrains in nonlinear dispersive systems. Proc. R. Soc. A 299 (1456), 59-76.

Benney, D. J. \& Roskes, G. J. 1969 Wave instabilities. Stud. Appl. Math. 48 (4), 377-385.

Boccotti, P. 1983 Some new results on statistical properties of wind waves. Appl. Ocean Res. 5 (3), 134-140.

Boccotti, P. 2000 Wave mechanics for ocean engineering. Elsevier.

Chawla, A. \& Kirby, J. T. 2002 Monochromatic and random wave breaking at blocking points. J. Geophys. Res. Oceans 107 (C7), 4-1.

Crawford, D. R., Lake, B. M., Saffman, P. G. \& Yuen, H. C. 1981 Stability of weakly nonlinear deep-water waves in two and three dimensions. J. Fluid Mech. 105, 177-191.

DAlzell, J.F. 1999 A note on finite depth second-order wave-wave interactions. Appl. Ocean Res. 21 (3), 105-111. 
Davey, A. \& Stewartson, K. 1974 On three-dimensional packets of surface waves. Proc. R. Soc. A 338, 101-110.

Dysthe, K., Krogstad, H . E. \& Müller, P. 2008 Oceanic rogue waves. Annu. Rev. Fluid Mech. 40, 287-310.

Dysthe, K. B. 1979 Note on a modification to the nonlinear schrödinger equation for application to deep water waves. Proc. R. Soc. A 369 (1736), 105-114.

Dysthe, K. B., Trulsen, K., Krogstad, H. E. \& Socquet-Juglard, H. 2003 Evolution of a narrow-band spectrum of random surface gravity waves. J. Fluid Mech. 478, 1-10.

Engsig-Karup, A. P, Bingham, H. B. \& Lindberg, O. 2009 An efficient flexible-order model for 3d nonlinear water waves. J. Comp. Phys. 228 (6), 2100-2118.

Ewans, K. C. 1998 Observations of the directional spectrum of fetch-limited waves. J. Phys. Oceanogr. 28 (3), 495-512.

Fadaeiazar, E., Alberello, A., Onorato, M., Leontini, J., Frascoli, F., Waseda, T. \& Toffoli, A. 2018 Wave turbulence and intermittency in directional wave fields. Wave Motion 83, 94-101.

Fedele, F. 2014 On certain properties of the compact Zakharov equation. J. Fluid Mech. 748, $692-711$.

Fedele, F., Brennan, J., León, S. P. De, Dudley, J. \& Dias, F 2016 Real world ocean rogue waves explained without the modulational instability. Sci Rep 6, 27715.

Fitzgerald, C. J., Taylor, P. H., Eatock Taylor, R. E., Grice, J. \& Zang, J. 2014 Phase manipulation and the harmonic components of ringing forces on a surface-piercing column. Proc. R. Soc. A 470, 105901.

Francius, M. \& Kharif, C. 2003 On the disappearance of the lowest-order instability for steep gravity waves in finite depth. Phys. Fluids 15 (8), 2445-2448.

Fujimoto, W., Waseda, T. \& WebB, A. 2019 Impact of the four-wave quasi-resonance on freak wave shapes in the ocean. Ocean Dyn. 69, 101-121.

GibBs, R. H. \& TAYLOR, P. H. 2005 Formation of walls of water in 'fully' nonlinear simulations. Appl. Ocean Res. 27, 142-157.

Gibson, R. S. \& Swan, C. 2007 The evolution of large ocean waves: the role of local and rapid spectral changes. Proc. R. Soc. A 463, 21-48.

Gramstad, O. \& Trulsen, K. 2007 Influence of crest and group length on the occurrence of freak waves. J. Fluid Mech. 582, 463-472.

Hara, T. \& MEI, C. C. 1991 Frequency downshift in narrowbanded surface waves under the influence of wind. J. Fluid Mech. 230, 429-477.

Hasselmann, K. 1962 On the non-linear energy transfer in a gravity-wave spectrum. Part 1: General theory. J. Fluid Mech. 12, 481-500.

Hasselmann, K. \& Others 1973 Measurements of wind-wave growth and swell decay during the Joint North Sea Wave Project (JONSWAP). Deutch Hydrogr. Z. A8, 1-95.

Hwang, P. A., Wang, D. W., Walsh, E. J., Krabill, W. B. \& Swift, R. N. 2000 Airborne measurements of the wavenumber spectra of ocean surface waves. Part ii: Directional distribution. J. Phys. Oceanogr. 30 (11), 2768-2787.

Janssen, P. A. E. M. 2003 Nonlinear four-wave interactions and freak waves. J. Phys. Oceanogr. 33, 863-884.

Janssen, P. A. E. M. \& Onorato, M. 2007 The intermediate water depth limit of the Zakharov equation and consequences for wave prediction. J. Phys. Oceanogr. 37 (10), 2389-2400.

Kharif, C., Giovanangeli, J. P., Touboul, J., Grare, L., \& Pelinovsky, E. $2008 a$ Influence of wind on extreme wave events: experimental and numerical approaches. J. Fluid Mech. 594, 209-247.

Kharif, C. \& Pelinovsky, E. 2003 Physical mechanisms of the rogue wave phenomenon. Eur. J. Mech. B/Fluids 22, 603-634.

Kharif, C., Pelinovsky, E. \& Slunyaev, A. $2008 b$ Rogue waves in the ocean. Springer Science \& Business Media.

Komen, G. J., Cavaleri, L., Donelan, M., Hasselmann, K., Hasselmann, S. \& Janssen, P. A. E. M. 1994 Dynamics and Modelling of Ocean Waves. Cambridge University Press.

Lake, B. M., Yuen, H. C., Rungaldier, H. \& Ferguson, W. E. 1977 Nonlinear deep-water waves: theory and experiment. part 2. evolution of a continuous wave train. J. Fluid Mech. 83 (1), 49-74. 
Latheef, M., Swan, C. \& Spinneken, J. 2017 A laboratory study of nonlinear changes in the directionality of extreme seas. Proc. R. Soc. A 473, 20160290.

Leckler, F., Ardhuin, F., Peureux, C., Benetazzo, A., Bergamasco, F. \& Dulov, V. 2015 Analysis and interpretation of frequency-wavenumber spectra of young wind waves. J. Phys. Oceanogr. 45 (10), 2484-2496.

Lindgren, G. 1970 Some properties of a normal process near a local maximum. Ann. Math. Stat 41 (6), 1870-1883.

Longuet-Higgins, M. S. 1976 On the nonlinear transfer of energy in the peak of a gravity-wave spectrum: a simplified model. J. Fluid Mech. 347, 311-328.

Longuet-Higgins, M. S. 1978 The instabilities of gravity waves of finite amplitude in deep water. ii. Subharmonics. Proc. R. Soc. A 360, 489-505.

Ma, Y., Dong, G., Perlin, M., Ma, X., Wang, G. \& Xu, J. 2010 Laboratory observations of wave evolution, modulation and blocking due to spatially varying opposing currents. J. Fluid Mech. 661, 108-129.

MCLean, J. W. $1982 a$ Instabilities of finite-amplitude gravity waves on water of finite depth. J. Fluid Mech. 114, 331-341.

McLean, J. W. $1982 b$ Instabilities of finite-amplitude water waves. J. Fluid Mech. 114, 315330.

Melville, W. K. 1982 The instability and breaking of deep-water waves. J. Fluid Mech. 115, $165-185$.

Meylan, M. H., Bennetts, L. G., Mosig, J. E. M., Rogers, W. E., Doble, M. J. \& Peter, M. A. 2018 Dispersion relations, power laws, and energy loss for waves in the marginal ice zone. J. Geophys. Res. Oceans 123 (5), 3322-3335.

Mori, N., Onorato, M. \& Janssen, P. A. E. M. 2011 On the estimation of the kurtosis in directional sea states for freak wave forecasting. J. Phys. Oceanogr. 41, 1484-1497.

Onorato, M., Osborne, A. R., Serio, M. \& Bertone, S. 2001 Freak waves in random oceanic sea states. Phys. Rev. Lett 86 (25), 5831.

Onorato, M., Osborne, A. R., Serio, M., Resio, D., Pushkarev, A., Zakharov, V. E. \& Brandini, C. 2002 Freely decaying weak turbulence for sea surface gravity waves. Phys. Rev. Lett 89 (14), 144501.

Onorato, M. \& Suret, P. 2016 Twenty years of progresses in oceanic rogue waves: the role played by weakly nonlinear models. Natural Hazards 84 (2), 541-548.

Paulsen, B. T., Bredmose, H., Bingham, H. B. \& Jacobsen, N. G. 2014 Forcing of a bottom-mounted circular cylinder by steep regular water waves at finite depth. J. Fluid Mech. 755, 1-34.

Perlin, M., Choi, W. \& Tian, Z. 2013 Breaking waves in deep and intermediate waters. Annu. Rev. Fluid Mech. 45, 115-145.

Peureux, C., Benetazzo, A. \& Ardhuin, F. 2018 Note on the directional properties of meter-scale gravity waves. Ocean Sci. 14 (1), 41-52.

PhILlips, O. M. 1960 On the dynamics of unsteady gravity waves of finite amplitude. Part 1. The elementary interactions. J. Fluid Mech. 9 (2), 193-217.

Rapizo, H., Waseda, T., Babanin, A. V. \& Toffoli, A. 2016 Laboratory experiments on the effects of a variable current field on the spectral geometry of water waves. J. Phys. Oceanogr. 46 (9), 2695-2717.

Segur, H., Henderson, D., Carter, J., Hammack, J., Li, C., Pheiff, D. \& Socha, K. 2005 Stabilizing the benjamin-feir instability. J. Fluid Mech. 539, 229-271.

Shemer, L., Sergeeva, A. \& Liberzon, D. 2010 Effect of the initial spectrum on the spatial evolution of statistics of unidirectional nonlinear random waves. J. Geophys. Res. Oceans 115, C12039.

Simanesew, A., Krogstad, H. E., Trulsen, K. \& Nieto Borge, J. C. 2016 Development of frequency-dependent ocean wave directional distributions. Appl. Ocean Res. 59, 304-312.

Simanesew, A., Krogstad, H. E., Trulsen, K. \& Nieto Borge, J. C. 2018 Bimodality of directional distributions in ocean wave spectra: A comparison of data-adaptive estimation techniques. J. Atmos. Ocean Tech. 35 (2), 365-384.

Socquet-Juglard, H., Dysthe, K., Trulsen, K., Krogstad, H. E. \& Liu, J. 2005 Probability distributions of surface gravity waves during spectral changes. J. Fluid Mech. 542, 195-216. 
Steer, J. N., Borthwick, A. G. L, Onorato, M., Chabchoub, A. \& van den Bremer, T. S. 2019 Hydrodynamic $X$ waves. Phys. Rev. Lett 123 (18), 184501.

Stiassnie, M. 1984 Note on the modified nonlinear Schrödinger equation for deep water waves. Wave Motion 6, 431-433.

Stiassnie, M. 2001 Nonlinear interactions of inhomogenous random water waves. In ECMWF report of the Workshop on Ocean Waves Forecasting, European Centre for Medium-Range Weather Forecasts, Reading, UK, pp.39-52.

Stiassnie, M. 2017 On the strength of the weakly nonlinear theory for surface gravity waves. J. Fluid Mech. 810, 1-4.

Stiassnie, M \& Shemer, L. 2005 On the interaction of four water-waves. Wave Motion 41, $307-328$.

Stuhlmeier, R. \& Stiassnie, M. 2017 Evolution of statistically inhomogenous degenerate water wave quartets. Proc. R. Soc. A 376, 20170101.

Su, M. Y., Bergin, M., Marler, P. \& Myrick, R. 1982 Experiments on nonlinear instabilities and evolution of steep gravity-wave trains. J. Fluid Mech. 124, 45-72.

Toffoli, A., Bennetts, L. G., Meylan, M. H., Cavaliere, C., Alberello, A., Elsnab, J. \& Monty, J. P. 2015 Sea ice floes dissipate the energy of steep ocean waves. Geophys. Res. 42 (20), 8547-8554.

Toffoli, A., Benoit, M., Onorato, M. \& Bitner-Gregersen, E. M. 2009 The effect of third-order nonlinearity on statistical properties of random directional waves in finite depth. Nonlinear Proc. Geoph. 16 (1), 131.

Toffoli, A., Onorato, M., Bitner-Gregersen, E. M. \& Monbaliu, J. 2010 Development of a bimodal structure in ocean wave spectra. J. Geophys. Res. Oceans 115, C03006.

Trulsen, K. 2018 Rogue waves in the ocean, the role of modulational instability, and abrupt changes of environmental conditions that can provoke non equilibrium wave dynamics. In The Ocean in Motion, pp. 239-247. Springer.

Trulsen, K. \& Dysthe, K. B. 1990 Frequency down-shift through self modulation and breaking. In Water wave kinematics, pp. 561-572. Springer.

Trulsen, K. \& Dysthe, K. B. 1996 A modified nonlinear Schrödinger equation for broader bandwidth gravity waves on deep water. Wave Motion 24 (3), 281-289.

Trulsen, K. \& Dysthe, K. B. 1997 Frequency downshift in three-dimensional wave trains in a deep basin. J. Fluid Mech. 352, 359-373.

Tulin, M. P. \& WASEDA, T. 1999 Laboratory observations of wave group evolution, including breaking effects. J. Fluid Mech. 378, 197-232.

Waseda, T., Kinoshita, T., Cavaleri, L. \& Toffoli, A. 2015 Third-order resonant wave interactions under the influence of background current fields. J. Fluid Mech. 784, 51-73.

Waseda, T., Toba, Y. \& Tulin, M. P. 2001 Adjustment of wind waves to sudden changes of wind speed. J. Oceanogr. 57, 519-533.

Whitham, G. B. 1967 Non-linear dispersion of water waves. J. Fluid Mech. 27 (2), 399-412.

Whitham, G. B. 1974 Linear and Nonlinear Waves. John Wiley and Sons.

XiaO, W., Liu, Y., Wu, G. \& Yue, D. K. P. 2013 Rogue wave occurrence and dynamics by direct simulations of nonlinear wave-field evolution. J. Fluid Mech. 720, 357-392.

Zakharov, V. E. 1968 Stability of periodic waves of finite amplitude on the surface of a deep fluid. J. Appl. Mech. Tech. Phys. 9, 190-194. 